\title{
Clinically-translatable High-fidelity Photoacoustic Tomography Enhanced by Virtual Point Sources
}

Junjie Yao ( $\square$ junjie.yao@duke.edu )

Duke University https://orcid.org/0000-0002-2381-706X

\section{Yuqi Tang}

Duke University

Shanshan Tang

Mayo Clinic

Chengwu Huang

Mayo Clinic https://orcid.org/0000-0003-0745-821X

Shigao Chen

Mayo Clinic

\section{Article}

Keywords: Photoacoustic tomography, deep imaging, virtual point source, microbubbles, single value decomposition, hemodynamic imaging, functional imaging

Posted Date: December 17th, 2021

DOI: https://doi.org/10.21203/rs.3.rs-1153472/v1

License: (c) (i) This work is licensed under a Creative Commons Attribution 4.0 International License.

Read Full License 


\title{
Clinically-translatable High-fidelity Photoacoustic Tomography Enhanced by Virtual Point Sources
}

\author{
Yuqi Tang ${ }^{1}$, Shanshan Tang², Chengwu Huang ${ }^{2}$, Shigao Chen², Junjie Yao ${ }^{1, *}$ \\ ${ }^{1}$ Photoacoustic Imaging Lab, Department of Biomedical Engineering, Duke University, \\ Durham, NC \\ 2 Ultrasound Imaging Lab, Department of Biomedical Engineering, Mayo Clinic, \\ Rochester, MN \\ ${ }^{*}$ Correspondence to junjie.yao@duke.edu
}

\begin{abstract}
Photoacoustic tomography (PAT), a hybrid imaging modality that acoustically detects the optical absorption contrast, is a promising technology for imaging hemodynamic functions in deep tissues. Particularly, PAT is capable of measuring the blood oxygenation level using hemoglobin as the endogenous contrast. However, the most clinically compatible PAT configuration usually employs a linear ultrasound transducer array and often suffers from the poor image fidelity, mostly due to the limited detection view of the transducer array. PAT can be improved by employing highly-absorbing contrast agents such as droplets and nanoparticles, which, however, have low clinical translation potential due to safety concerns and regulatory hurdles. Moreover, unlike hemoglobin, these exogenous contrast agents cannot report the functional hemodynamic information. In this work, we have developed a new methodology that can improve PAT's image fidelity without hampering its functional capability or clinical translation potential. By using clinicallyapproved microbubbles as virtual point sources that strongly scatter the local pressure waves generated by surrounding hemoglobin, we can overcome the limited-detectionview problem and achieve high-fidelity functional PAT in deep tissues, a technology referred to as virtual-point-source PAT (VPS-PAT). We have thoroughly investigated the working principle of VPS-PAT by numerical simulations and phantom validations, showing the acoustic origin of signal enhancement and the superiority over traditional PAT. We have also demonstrated proof-of-concept applications of functional VPT-PAT for in vivo
\end{abstract}


small-animal studies with physiological challenges. We expect that VPS-PAT can find broad applications in biomedical research and accelerated translation to clinical impact.

Keywords: Photoacoustic tomography, deep imaging, virtual point source, microbubbles, single value decomposition, hemodynamic imaging, functional imaging

\section{Main}

Photoacoustic tomography (PAT, also referred to as optoacoustic tomography) is a hybrid imaging modality that acoustically detects the optical absorption contrast via photoacoustic (PA) effect. PAT has been proven powerful for imaging hemodynamics using hemoglobin as the endogenous contrast in biological tissues, with high resolution, deep penetration, and inherent functional and molecular sensitivity. Using diffuse light excitation and acoustic detection, PAT is less limited by the tissue's strong optical scattering, and can achieve high spatial resolution and large penetration depth that are not achievable by pure optical imaging [1], [2]. Recently, PAT has been increasingly applied in (pre)clinical studies such as breast cancer screening [3]-[10], skin disease diagnosis [11]-[14], and functional brain imaging [15]-[23]. So far, most clinicallytranslatable PAT technologies utilize linear ultrasound transducer arrays, which are convenient for different anatomical locations and directly compatible with clinical ultrasound scanners. However, linear-array-based PAT systems are often hindered by the limited detection view problem, which manifests as low fidelity in the reconstructed images, such as missing vertical vessels, reduced spatial resolution, and strong streaking artifacts [24]-[26].

The limited view problem arises from the coherent signal generation in the PA effect, as well as the high density of red blood cells in the blood volume $(\sim 50 \% \mathrm{v} / \mathrm{v})$ and thus the homogeneity of optical absorption. When illuminated with pulsed laser, each individual red blood cell generates PA signals with coherent phases. With a high red blood cell density, pressure waves from the neighboring red blood cells tend to cancel out and constructive interference is only prominent at the vessel boundaries [27], [28]. Therefore, instead of emitting spherical waves from each individual red blood cells, the blood vessels generate pressure waves traveling along the vessel boundaries, which have high 
dependence on the vessel orientations. For an exact image reconstruction, PAT requires full record of the pressure wavefront travelling in all orientations, i.e., a solid angular detection aperture of $2 \pi$ for a planar detection geometry or $4 \pi$ for a spherical or a cylindrical detection geometry [24], [29]. When a linear transducer array with limited detection aperture is used, only partial wavefronts from the blood vessels can be recorded, which results in the limited view problem.

Current solutions for the limited view problem in PAT include increasing the detection aperture, employing exogenous highly-absorbing contrast agent, and reconstructing with deep learning approaches. Increasing the detection aperture is usually achieved by either rotating the linear transducer array to cover a large detection angle [30], [31], or using a ring-shaped or spherical transducer [32], [33]. The former method requires complex system design, and the later greatly increases the cost of the system. Both methods would substantially hamper the clinical translation. The limited view problem can also be addressed by introducing sparsely-distributed exogenous contrast agents such as gold nanoparticles, which have much stronger optical absorption than hemoglobin and can disrupt the homogeneity of the optical absorption inside the bloodstream. The exogenous contrast agents can generate spherically travelling pressure waves and be well detected by the linear transducer array. Blood vessels in arbitrary orientations can be imaged by accumulating sufficient number of frames [28], [34]. The major limitation of this approach is that the received PA signals are from the exogenous absorbers, and thus physiological information such as oxygen saturation of hemoglobin $\left(\mathrm{sO}_{2}\right)$ cannot be estimated. In addition, none of these exogenous contrast agents are approved by FDA for clinical applications with unknown safety effects and difficult regulatory clearance. Alternatively, various deep-learning networks have been used for artifact removal in linear-array based PAT, which have shown improved image fidelity [35]-[41]. However, the deep-learning based methods may introduce overfitting artifacts for data with significant noise, and their in vivo performance are mostly unknown [42]. Therefore, there still lacks a clinicallytranslatable PAT technology with high imaging quality and functional imaging capability. In this work, we have demonstrated a novel method to overcome the limited view problem and improve the image fidelity of clinically-translatable PAT, by using microbubbles as 
virtual point sources (VPS), referred to as VPS-PAT. In VPS-PAT, although the microbubbles themselves have close to zero optical absorption, they can redirect the local PA pressure waves travelling in any directions via Rayleigh scattering and emit spherical waves that can be well detected by the linear transducer array. By reconstructing the sparsely distributed microbubbles as VPS over a number of imaging frames, VPS-PAT can produce high-fidelity images of the vasculature with improved visibility of the vertical structures and reduced reconstruction artifacts. Importantly, since the detected signals from the VPS originate from the surrounding hemoglobin, VPS-PAT keeps the functional sensitivity to the blood oxygenation level. Moreover, since microbubbles have been routinely used in clinical ultrasound imaging, we expect that VPS-PAT should have accelerated clinical translation compared with other PAT technologies relying on highlyabsorbing contrast agents. We have thoroughly investigated the working principle of VPSPAT by numerical simulations and phantom studies, showing its superior imaging performance over traditional PAT. We have applied VPS-PAT for small animal studies to demonstrate its functional imaging capability. Our experimental results have collectively illustrated that VPS-PAT can effectively improve the image fidelity of clinically-translatable PAT, and may find broad applications in preclinical research and clinical practice.

\section{Results}

\section{Imaging principle of VPS-PAT}

FDA-approved microbubbles are usually made of a gas core (e.g., nitrogen or perfluorocarbon gases) encapsulated by a solid shell (e.g., lipid or protein). Microbubbles have been widely used as contrast agents in clinical ultrasound imaging, in which they can enhance the blood vessel signals by strongly scattering the transmitted ultrasound waves [43]-[46]. Typically, native microbubbles without being conjugated with other chromophores cannot be used as contrast agents for PAT, because microbubbles have negligible optical absorption compared to hemoglobin (Supplementary Fig. 1). Unlike the highly-absorbing nanoparticles and droplets, microbubbles may serve as negative contrast agents in the bloodstream and break the homogeneity of the optical absorption. However, using numerical simulations, we have demonstrated that, due to the small size $(\sim 5 \mu \mathrm{m})$ and sparsity of the microbubbles $\left(4 \times 10^{6}\right.$ microbubbles $\left./ \mathrm{ml}\right)$, the zero optical 
absorption of microbubbles has practically negligible impact on the generated PA signals and thus has little contribution to mitigating the limited view problem (Supplementary Fig. 2) [47], [48].

However, since microbubbles have large acoustic impedance mismatch with the surrounding bloodstream [49], we expect that the strong acoustic scattering of microbubbles can play a much more important role in addressing the limited view problem in PAT. The most common PAT system usually uses a linear array transducer (Fig. 1a). The linear array transducer typically has a central frequency between $2-10 \mathrm{MHz}$, corresponding to an in vivo acoustic wavelength of 150-750 $\mu \mathrm{m}$ [50]. Microbubbles used for clinical applications typically have a diameter between 0.5 and $10 \mu \mathrm{m}$, which is much smaller than the PA pressure wavelengths and thus can be considered as point targets [49]. Similar to contrast-enhanced ultrasound imaging, sparsely distributed microbubbles can function as Rayleigh scatterers and redistribute the surrounding PA pressure waves travelling in all directions (Fig. 1b, Supplementary Fig. 3). Because Rayleigh scattering has low anisotropy (or directivity dependence), the redirected PA waves by microbubbles are close to spherical waves, and thus can be readily detected by the linear transducer array positioned at any orientation. By filtering out the static PA signals directly from the blood via single value decomposition (SVD), VPS-PAT detects only the scattered PA signals from the microbubbles, and then reconstructs the images of the microbubbles as virtual point sources (Fig. 1c). By accumulating the microbubble signals from multiple independent frames, VPS-PAT is able to reconstruct the otherwise invisible vessel structures and thus improve the image fidelity.

\section{Simulation of VPS-PAT using microbubbles as virtual point sources}

To demonstrate the concept of VPS-PAT, we performed 2D k-wave simulation of PA imaging of a vertical blood vessel inside a mouse liver model [51]. The simulation covered a field of view of $12 \times 15 \mathrm{~mm}^{2}$ region that included skin, muscle, fat, and liver, with the linear array transducer placed on top of the skin surface. A vertical blood vessel $(5 \mathrm{~mm}$ length, $0.2 \mathrm{~mm}$ diameter) was placed $11 \mathrm{~mm}$ from the transducer surface (Fig. 2a). The acoustic properties were heterogeneous across different tissue types in speed of sound, tissue density, and acoustic attenuation coefficient (Supplementary Fig. 4) [52], [53]. We 
assumed that optical fluence was homogenous around the blood vessel and hemoglobin was the dominating light absorber. A time-reversal based method was used to reconstruct PA images from the simulated PA data [54]. The image reconstruction also assumed homogenous tissue density and sound speed. The microbubbles had a diameter of $5 \mu \mathrm{m}$, an optical absorption coefficient of zero, and an acoustic impedance of $500 \mathrm{~kg} /\left(\mathrm{m}^{2} \mathrm{~s}\right)$.

Without any microbubbles inside the vertical vessel (Figs. 2b), the forward simulation shows that the generated PA pressure waves propagated sideways along the vessel (Supplementary Video 1). Thus, only the top and bottom boundaries of the vessel were visible in the reconstructed PA image, while the middle portion between the two boundaries was missing, demonstrating the classic limited view problem (Fig. 2c). With a single static microbubble inside the vessel (Fig. 2d), the PA signals redirected by the bubble travelled as a spherical wave and was detected by the transducer array (Supplementary Video 2). The reconstructed image clearly shows the microbubble as a point source at the correct position (Fig. 2e). Similarly, with five static microbubbles sparsely distributed inside the vessel (Fig. 2f, Supplementary Video 3), their positions were all correctly reconstructed in the PA image (Fig. $\mathbf{2 g}$ ), showing partially the vertical vessel lumen. We further analyzed the raw PA signals detected by the transducer in the three different scenarios, and the results show that the scattered signals by the microbubbles were $\sim 3-4$ times weaker than the boundary signals (Figs. 2h). Consequently, in the reconstructed images, the microbubbles as the virtual points sources were also $\sim 3-4$ times weaker than the vessel boundaries (Fig. 2i). Nevertheless, the microbubbles enabled better visualization of the vessel lumen with a 3-fold increase in the contrast to noise ratio (CNR) with one microbubble, and a 5 -fold increase with five microbubbles (Fig. 2j) [55], [56].

We then simulated VPS-PAT with flowing microbubbles in the bloodstream. The flowing microbubbles were sparsely and randomly distributed in blood vessels. When a blood vessel was imaged over multiple frames with a relatively low frame rate, each frame had a different set of microbubble locations. By accumulating a certain number of frames and merging the reconstructed locations of all virtual point sources, we can recover the full vertical vessel structure that is otherwise invisible in traditional PAT. To mimic 
microbubbles flowing in bloodstream, we simulated and reconstructed 20 different frames. Each frame had five microbubbles defined at random locations within the vertical blood vessel (Fig. 2a), which corresponded to a concentration of $2.2 \times 10^{6}$ particles $/ \mathrm{ml}$ or $\sim 0.03 \%$ volume concentration. This concentration was slightly lower than the typical clinical dose in contrast-enhanced ultrasound imaging [47].

As the scattered signals from the virtual point sources were weaker than the nonscattered signals from the vessel boundaries, we investigated different signal processing techniques to suppress the non-scattered signals. We first applied singular value decomposition (SVD) to the reconstructed images and removed high singular values with an empirically optimized cutting threshold (Fig. 3a). The non-scattered signals from the top and bottom boundaries were effectively filtered out due to the high temporal coherence over different frames. Compared to the original B-mode images (Figs. 3c-d), the scattered signals from the VPSs were largely preserved, because of their low temporal coherence from frame to frame (Figs. 3e-f). By merging the SVD-filtered images and accumulating all the reconstructed VPSs as the final image, the visibility of the vertical vessel as well as the CNRs were greatly improved (Figs. 3i-k). We also explored another signal processing method by applying a high-pass filter on the RF data across consecutive frames (Fig. 3b), which highlighted the scattered signals from the VPSs (Fig. 3g). The high-pass-filtered RF data was then reconstructed to form the images of the VPSs, revealing similar CNR improvements as the SVD-filtered image (Figs. $3 \mathbf{h}-\mathbf{k}$ ). However, the high-pass-filter-based image presented more reconstruction artifacts than the SVD-filtered image, likely because the SVD method was more tolerant to the multiscattering signals by considering the signal's spatial coherence as well.

\section{VPS-PAT for improving visibility of vertical structures}

We validated the concept of VPS-PAT by imaging a loose knot made of a silicone capillary tube with a $300-\mu \mathrm{m}$ inner diameter. The knot was embedded in $1 \%$ transparent agar and aligned with the linear transducer's imaging plane. We delivered pulsed laser excitation at $532 \mathrm{~nm}$ with a pulse energy of $7 \mathrm{~mJ}$ through the linear bifurcated light guide (Fig. 4a). Whole bovine blood was mixed with microbubbles to yield a final concentration of $2 \times 10^{6}$ microbubbles $/ \mathrm{ml}$. The blood-microbubble mixture was perfused into the capillary knot with 
a flow speed of $20 \mathrm{~mm} / \mathrm{s}$, using a syringe pump to mimic blood flow (Fig. $4 \mathbf{b}$, Supplementary Video 4). A total of 500 consecutive frames were acquired with a frame rate of $10 \mathrm{~Hz}$. As a control, we also imaged whole bovine blood without microbubbles (Fig. 4c, Supplementary Video 5). The same SVD filter was applied to both experiments. The US Doppler imaging was also performed on the same structure as a reference (Supplementary Video 6).

As the knot phantom had both horizontal and vertical segments, it was an ideal target for demonstrating the effectiveness of VPS-PAT in visualizing arbitrary target orientation. In the B-mode PA image, the vertical segments of the knot were poorly visible, as scattered signals from the microbubbles were overshadowed by non-scattered signals (Fig. 4b). As expected, the SVD filter was able to suppress the non-scattered signals directly from hemoglobin and recover the correct shape of the knot (Fig. 4d, Supplementary Video 7). By contrast, without the microbubbles, neither the B-mode image nor the SVD-filtered image was able to recover the vertical segments (Figs. 4c and 4e, Supplementary Video 8). The knot structure recovered by VPS-PAT was confirmed by the US Doppler image (Fig. 4f). We also extracted the image intensity profiles in both lateral and axial directions of the SVD-filtered images. With microbubbles, vertical sections of the knot were clearly distinguished from the background (Figs. $\mathbf{4 g - h}$ ). CNR was calculated at four representative regions, and microbubbles helped improve the CNR for both horizontal and vertical regions of the knot (Fig. 4i). This phantom experiment shows that microbubbles as VPS were able to improve the visibility of the vertical structures, and thus mitigate the limited view problem in PAT.

\section{VPS-PAT for measuring oxygenation of hemoglobin $\left(\mathrm{sO}_{2}\right)$}

In addition to improving the visibility of vertical structures, the scattered PA signals from the microbubbles preserve the functional information of the surrounding hemoglobin and $\mathrm{SO}_{2}$ can be estimated with multispectral PA imaging, which is fundamentally different from other highly-absorbing exogenous contrast agents. To demonstrate the functional sensitivity of VPS-PAT, we prepared fully oxygenated and deoxygenated bovine blood by adding sodium bicarbonate ( $37 \mathrm{mg} / \mathrm{ml}$ of blood, followed by 5 -minute $100 \% \mathrm{O}_{2}$ infusion) and sodium dithionite $\left(2.5 \mathrm{mg} / \mathrm{ml}\right.$ of blood, followed by 5 -minute $100 \% \mathrm{CO}_{2}$ infusion), 
respectively (Fig. 5a) [57], [58]. The oxygenated and deoxygenated blood were expected to have nearly $100 \%$ and $0 \% \mathrm{sO}_{2}$ respectively. The oxygenated and deoxygenated blood samples were then mixed with microbubbles and perfused into a capillary tube with a constant flow speed of $20 \mathrm{~mm} / \mathrm{s}$ (Fig. $\mathbf{5 b}$ ). The capillary tube was imaged by VPS-PAT with dual-wavelength excitation at 532 and $1064 \mathrm{~nm}$ (Figs. 5c-f). The $\mathrm{sO}_{2}$ image was calculated from both the B-mode and the SVD-filtered images using the linear spectral unmixing method [59]-[61]. Here, we did not consider the spectral coloring effect in the transparent agar phantom [62]. The results clearly show that the B-mode PA images were not able to recover the $\mathrm{sO}_{2}$ levels in the vertical segments of the phantom (Figs. $5 \mathrm{c}$ and 5e). By contrast, VPS-PAT was able to accurately quantify the $\mathrm{sO}_{2}$ levels of the blood phantoms (Figs. $\mathbf{5 d}$ and $\mathbf{5 f}$ ). It is worth noting that the $\mathrm{sO}_{2}$ estimation by VPS-PAT was consistent at all segments along the capillary tube.

\section{VPS-PAT for in vivo rat liver imaging under hypoxia challenge.}

To validate the in vivo performance of VPS-PAT, we imaged the liver vasculature of a rat under normoxia and hypoxia. We placed the rat in the prone position on the imaging window, with the laser light illumination $(1064 \mathrm{~nm})$ and the linear transducer array immersed in coupling medium underneath the imaging window (Fig. 6a). The same tissue plane around the liver region was imaged before and after microbubble injection. A total of 1000 frames were acquired with a frame rate of $50 \mathrm{~Hz}$ and a data acquisition time of 20 seconds. The reconstructed PA images were first stabilized to eliminate the respiration motion artifacts, and then processed with the SVD filter to extract the scattered signals from the microbubbles. Here, we used a dual speed-of-sound (SOS) image reconstruction method, because of the relatively large difference in SOS between the coupling medium and the rat tissue (Supplementary Fig. 5). The dual SOS image reconstruction was able to better converge the relatively weak PA signals scattered from microbubbles.

The results have clearly demonstrated that VPS-PAT was able to image more blood vessels with improved contrast and reduced artifacts, when compared with the B-mode PA images (Figs. 6b-c). The VPS-PAT image showed similar anatomical structure as the Doppler ultrasound image (Fig. 6d) and revealed clutters of small vessels as well as vertical vessels inside the liver region, with a penetration depth of $\sim 1 \mathrm{~cm}$ (Figs. 6e-f). In 
order to highlight the necessity of microbubbles, we also tested the imaging process on the same animal without the microbubbles. In this case, the flowing blood may still provide some PA signal fluctuations over multiple frames, due to the rearrangement of red blood cells from frame to frame [63]. Without microbubbles, the SVD process had reduced effect on improving the image quality (Supplementary Fig. 6), with weaker CNR and no major vessels beyond $5 \mathrm{~mm}$ depth.

To demonstrate the capability of functional imaging with VPS-PAT, we conducted hypoxia challenge on the rat and monitored the hemodynamic response around the liver region. Here, we used a single wavelength at $1064 \mathrm{~nm}$ for the relative oxygenation measurement, because deoxy-hemoglobin has a 20 times lower absorption coefficient at $1064 \mathrm{~nm}$ than that of oxygen-hemoglobin. The PA signal change at $1064 \mathrm{~nm}$ should mostly reflect the relative change in the oxy-hemoglobin concentration. We alternated the normoxia and hypoxia states for three cycles, with 1000 frames acquired for each condition in each cycle (Fig. 7a). The hypoxia challenge induced a systemic decrease in blood oxygenation level, and therefore, we observed a clear decrease in total PA signal and the vessel visibility, when compared to the normoxia conditions (Figs. 7b-c, Supplementary Fig. 7, Supplementary Videos 10). Again, the results show that the SVD-filtered images could visualize more vessels at larger depths under both conditions than the B-mode images, highlighting the fact that the SVD filtered signals were redirected from the surrounding hemoglobin by the microbubbles. We further analyzed regional signal intensities for vessel clutters (Fig. 7d) and signal profiles for individual vessels (Fig. 7e). The results showed that hypoxia led to a $>65 \%$ drop in the overall PA signal intensity at $1064 \mathrm{~nm}$, reflecting a decrease in the blood oxygenation level. We also quantified the relative signal changes from normoxia to hypoxia in the B-mode images (Fig. 7f) or SVD-filtered images (Fig. $\mathbf{7 g}$ ). The different signal changes under hypoxia challenge were indicative of the original blood oxygenation level under normoxia condition [64].

\section{Discussion}

We have developed a novel imaging technology VPS-PAT that uses acousticallyscattering microbubbles to improve the image fidelity of clinically-translatable PAT with a linear transducer array. Microbubbles are FDA approved contrast agent for clinical 
ultrasound imaging and are ideal Rayleigh scatterers of the PA pressure waves, due to their subwavelength sizes and large acoustic impedance mismatch from the surrounding blood. Disregarding the vessel orientation or shape, microbubbles can scatter and redirect the local PA signals towards the linear transducer array. The approximately isotropic scattering by the microbubbles effectively creates virtual point sources with spherical signal emission. For vertical vessel structures with PA pressure waves that are otherwise not detected by the linear transducer array, they can now be imaged by reconstructing the scattered PA signals. It is worth emphasizing that the acoustic scattering of the microbubbles is the dominating mechanism in VPS-PAT for the enhanced PA signals. In fact, the clinically-approved microbubbles have no optical absorption. Because of the small size and sparse distribution of the microbubbles in the bloodstream, their optical properties have negligible impact on the PA signal generation and propagation.

VPS-PAT uses microbubbles as virtual point sources, which has clear advantages over other methods using highly-absorbing exogenous contrast agents. Microbubbles are FDA approved for clinical use, which may accelerate the clinical translation of VPS-PAT. Moreover, we have demonstrated that, through both in vitro and in vivo experiments, the scattered signals by microbubbles can preserve the functional information from the surrounding blood, allowing measurement of important physiological parameters such as $\mathrm{sO}_{2}$. Nevertheless, in the reconstructed PA images, the microbubbles as virtual point sources, have 3-4 times weaker signals than the non-scattered signals directly from blood. We applied SVD filtering to remove the strong non-scattering signals to improve the CNR of virtual point sources. The SVD-filtered images show improved structure visibility and reduced limited-view artifacts, both of which are due to the spherical waves scattered by microbubbles as virtual point sources.

One important limitation of the current VPS-PAT system is that multiple frames are needed to accumulate enough scattered signals from microbubbles, which leads to a relatively long data acquisition time and thus respiration-induced motion artifacts for in vivo studies. In our current setup, the imaging speed is further limited by the low pulse repetition rate of $10-50 \mathrm{~Hz}$. We have shown that, with as few as 200 stabilized frames 
and a total acquisition time around 4-6 seconds, the visibility of major vessels and small vessel clusters were already improved compared to the B-mode images (Supplementary Fig. 8). Incorporating more frames could help improve the SNR of SVD-filtered image with more averaging, but also introduced respiration-induced artifacts that led to blurring of small features. One improvement of VPS-PAT is the incorporation of laser sources with a higher PRF to improve the imaging speed. High imaging speed will also significantly reduce the motion susceptibility, and may even allow tracking VPS to measure the blood flow speed [28], [65]. As our method relies on the relatively weak PA signal scattered by microbubbles, high SNR would help separate the VPS from the background. In deep tissues with low optical fluence and thus low SNR, advanced optical acoustic detection method with improved detection sensitivity may be applied [66]-[68].

For image reconstruction, we adapted a dual-SOS based method that was superior over the traditional single-SOS based method (Supplementary Figure 5). Although the dualSOS method did not significantly improve the B-mode PA images, it was critical for improving the SVD-filtered VPS images that were sensitive to the signal convergence of point targets. Nevertheless, we still assumed all types of tissues had the same speed of sound, which was not accurate especially for tissues with high fat content and fibers [52]. A more accurate reconstruction method with tissue-dependent speed of sound, such as fast marching method, can help improve the VPS signal convergence and thus further improve the detection sensitivity and SVD-filtered imaging quality [69]. Moreover, instead of global SVD filtering, block-wise SVD filtering with adaptive cutoff values will improve the visibility of small vessels as well as noise suppression [70].

Compared with previous methods using ring-shaped or spherical transducer arrays, our method using microbubbles as virtual point sources has much lower cost and computational burden. Compared to exogenous contrast agent for improving structure visibility, microbubbles have negligible optical absorption. Microbubbles only redirect the signals from the surrounding blood and thus preserve the functional information, which allows estimation of physiological parameters such as $\mathrm{sO}_{2}$ and blood flow speed. Microbubbles act as virtual point sources, which potentially allow super-resolution imaging via high-precision localization. Most importantly, as microbubbles are FDA 
approved contrast agents for US imaging, our approach is more clinically translatable than other preclinical contrast agents.

In summary, we expect that our new method VPS-PAT can provide high-fidelity hemodynamic information for preclinical and clinical applications such as disease monitoring, drug assessment, and surgical guidance.

\section{Acknowledgements}

We thank Dr. Caroline Connor for editing the manuscript. This work was sponsored by National Institutes of Health (R01 EB028143, R01 NS111039, RF1 NS115581, R21 EB027304, R21EB027981, R43 CA243822, R43 CA239830, R44 HL138185); American Heart Association Collaborative Sciences Award (18CSA34080277); Chan Zuckerberg Initiative Grant on Deep Tissue Imaging $2020-226178$ by Silicon Valley Community Foundation. The authors would like to thank Dr. Pengfei Song for the useful discussion and the engineering team at Sonovol for the technical support.

\section{Author contributions}

J.Y conceived and directed the project. Y.T. set up the imaging system, performed all experiments, and analyzed the data. S.T., C.H. and S.C. contributed to the data analysis and ultrasound Doppler imaging. Y.T. and J.Y. wrote the manuscript. All authors discussed the results and approved the writing.

\section{Competing interests}

The authors declare no competing interests.

\section{Additional information}

Supplementary information is available for this paper. 


\section{Methods}

\section{Photoacoustic tomography}

In PAT, photons in pulsed laser are absorbed by chromophores in tissue, and their energy is completely or partially converted to heat. The transient temperature rise leads to increased pressure and subsequent sound wave generation. The amplitude of pressure increase, as well as the intensity of the sound wave, is directly proportional to the chromophore concentration and local optical fluence. The sound wave is then detected by an ultrasonic transducer and used to form an image to tomographic map the original energy deposition in the tissue. Using acoustic detection, the penetration depth of PAT is less limited by optical scattering and is comparable to that of ultrasound imaging.

\section{VPS-PAT system}

The VPS-PAT system was adapted from a commercial ultrasound scanner (Vantage 256, Verasonics). An L7-4 linear array transducer (Philips ATL) was used for both phantom and animal studies. The transducer had 128 elements, with a central frequency of $5 \mathrm{MHz}$ and a $60 \%$ bandwidth. Laser pulses, available at 532 and $1064 \mathrm{~nm}$, with $8 \mathrm{~ns}$ pulse-width and pulse rep rate of $10 \mathrm{~Hz}$ (Q-smart 850, Quantel Laser) or $100 \mathrm{~Hz}$ (DRL 100, Qutantel Laser), were delivered with a bifurcated linear light guide (Dolan-Jenner, 50\% coupling efficiency) attached on the sides of the transducer and illuminated the elevational focal region of the transducer ( $\sim 25 \mathrm{~mm}$ focal length; $\sim 1 \mathrm{~mm}$ imaging plane thickness). The $1064 \mathrm{~nm}$ light for in vivo imaging had an energy fluence of $75 \mathrm{~mJ} / \mathrm{cm}^{2}$ on the sample surface, which was way below the ANSI laser safety limit. The channel data from the array transducer was sampled at $60 \mathrm{MHz}$ and saved for further processing.

\section{Ultrasound Doppler imaging}

The same commercial ultrasound scanner (Vantage 256, Verasonics, Washington) and the L7-4 linear array transducer were used to acquire in-phase/quadrature (IQ) data for both real-time B-mode and ultrafast Doppler imaging of microbubbles. US plane waves were transmitted at a center frequency of $5 \mathrm{MHz}$ with a PRF of $5000 \mathrm{~Hz}$. Coherent spatial compounding was implemented with 7 steering angles ( -3.5 degree to 3.5 degree with an increment of 1.16 degree), which led to a post-compounding frame rate of $500 \mathrm{~Hz}$. For a 
single frame, each transmission event was separated by $80 \mu$ s to cover an imaging depth of $35 \mathrm{~mm}$. An ensemble of 4000 compounded frames at the region of interest was acquired in 8 seconds and the IQ data were saved. The image data were stabilized using phase correlation-based sub-pixel registration [71]. Global singular value thresholding (SVT) was applied to the motion-corrected data to separate the microbubble signals from the tissue signals. As tissue signals typically resided in the large spectral components with high singular values, the SVT cutoff was determined by searching for the turning point which the singular value curve began to flatten [72]. The singular values larger than the cutoff were set to zero, and the ensemble after thresholding were averaged for further SNR improvement.

\section{k-Wave simulation}

The k-Wave toolbox was used for simulation [51]. In 2D k-Wave simulation, the field of view covered a $12 \times 15 \mathrm{~mm}^{2}$ with a grid size of $5 \times 5 \mu \mathrm{m}^{2}$. The microbubbles' location was randomly generated within the blood vessel region and was defined as a single pixel with acoustic impedance of $500 \mathrm{~kg} /\left(\mathrm{m}^{2} \mathrm{~s}\right)$. As the initial pressure in PAT is always smaller than $10 \mathrm{kPa}$, the microbubble oscillation is negligible, and we assumed no harmonics were generated. The sensor defined in the simulation had the same configuration as the L7-4

linear array, which contained 42 elements and $1 / 3$ of the original aperture. The acquired PA channel signal was filtered using a $5^{\text {th }}$-order Butterworth filter with a 4-7 MHz cutoff. As the pressure generated during the PA imaging is usually less than $10 \mathrm{kPa}$, the oscillation and the diameter change of the microbubble was negligible [73].

\section{Microbubble preparation}

Perfluorocarbon-filled phospholipid microbubbles (VesselVue, SonoVol) with mean diameter of $4.37 \mu \mathrm{m}$ were used for PAT. The vial had an initial concentration of $2.21 \times$ $10^{9}$ particles $/ \mathrm{ml}$ and was mixed with PBS to yield a final concentration of $2 \times 10^{6}$ and $2 \times$ $10^{7}$ particles/ml for phantom and animal study respectively. A 27 -gauge needle was used for injection via the tail vein to avoid microbubble bursting.

\section{Singular value decomposition filter}


Similar to slow-time signal in the US color Doppler image, the slow-time signal from PAT with microbubbles is comprised of three constituent components: 1) non-scattered signals, which are PA signals transmitted directly from blood and received within the detection aperture; 2) scattered signals, which are PA signals isotropically scattered from the microbubbles and received within the detection aperture; 3) random white noise, which originates from thermal and electronic noises [74]. For an image pixel that does not contain microbubbles, the slow-time signal should have only non-scattered signals and random white noise. A slow-time signal ensemble $\mathbf{x}$ can be expressed in following two forms for a give ensemble size ND:

$\mathbf{x}=\left[\begin{array}{c}x(0) \\ x(1) \\ \vdots \\ x\left(N_{D}-1\right)\end{array}\right]=\left\{\begin{array}{lr}\mathbf{d}+\mathbf{s}+\mathbf{n} \quad \text { (if with microbubbles) } \\ \mathbf{d}+\mathbf{n} & \text { (if without microbubbles) }\end{array}\right.$

In (1), $\mathbf{x}(\mathrm{n})$ represents the $\mathrm{n}^{\text {th }}$ slow-time data sample, and $\mathbf{d}, \mathbf{s}, \mathbf{n}$ are vectors of length $\mathrm{N}_{D}$ for non-scattered PA signals, scattered PA signals, and white noise, respectively. Based on our simulation results, the non-scattered signal, usually from horizontal structures, is stronger than other signal components, including scattered PA signals from both vertical and horizontal structures. Therefore, we need to suppress the non-scattered component.

It is known that eigenvalues contain information about the signal energy represented by the individual eigen-components. In particular, a large eigenvalue signifies that its respective eigen-component accounts for a substantial energy portion of the slow-time ensemble. Such a representation matches the characteristics of non-scattered PA signals. In order to use eigenvalue-based filtering, we first decomposed the slow-time signal contents into a sum of mutually orthogonal components that have the minimum meansquared modeling error. For a given slow-time ensemble $\mathbf{x}$ [75]-[77]:

$\boldsymbol{x} \approx \sum_{k} \gamma_{k} \mathbf{e}_{k} .(2)$

$\mathbf{e}_{k}$ is the $k^{\text {th }}$ eigenvector and $\gamma_{k}$ is the corresponding expansion weight that satisfies the following orthogonality relation:

$E\left\{\left(\gamma_{k} \mathbf{e}_{k}\right)^{* T}\left(\gamma_{k} \mathbf{e}_{k}\right)\right\}=\left\{\begin{array}{c}\lambda_{k}(k=l) \\ 0(k \neq l)\end{array}\right.$. 
By identifying the eigen-components of non-scattered signals as the ones whose eigenvalues are above a predefined threshold $\lambda_{d}$, we can remove these eigencomponents and suppress the non-scattered signals. The resultant eigen-component subset for scattered siganls is

$\Phi_{s}=\left\{\mathbf{e}_{k} \mid \lambda_{k}<\lambda_{d}\right\}$. (4)

For our application, the threshold $\lambda_{d}$ was empirically set relative to the largest eigenvalue as $\lambda_{d} \approx 0.1 \lambda_{\max }$.

\section{Experimental animals}

Female Wistar rats (Charles River Laboratories:150 - 200g, 6-10 months old) were used for the in vivo studies. The laboratory animal protocol was approved by the Institutional Animal Care \& Use Committee of Duke University. During the experiment, the rat's temperature was kept at $37^{\circ} \mathrm{C}$ by a heating lamp. Inhalant isoflurane was used for anesthesia with $5 \% \mathrm{v} / \mathrm{v}$ for induction and $2 \% \mathrm{v} / \mathrm{v}$ for maintenance. Compressed air flow rate through the facemask was set to $1000 \mathrm{~mL} / \mathrm{min}$, and the breathing rate of rat was around 55-60 times/min. Rat's fur at the liver region was removed with shaving cream. The rat was placed in prone position on the acoustically and optically transparent imaging window and secured with surgical tape. A dose of $100 \mu \mathrm{L}$ microbubbles $\left(2 \times 10^{7}\right.$ particles $/ \mathrm{ml}$ ) was injected via the tail vein before each data acquisition session. Each data acquisition session was separated at least 10 minutes apart to ensure that injected bubbles had been cleared out. Each injection corresponded to approximately 170,000 microbubbles per $\mathrm{mL}$ of blood, which was lower than the typical dose in clinical practice.

\section{Hypoxia challenge}

The rats were housed and anesthetized under normoxia $\left(21 \% \mathrm{O}_{2}\right)$. During the hypoxia challenge, isoflurane vaporizer was constantly supplied with a mix of compressed air and pure $\mathrm{N}_{2}$ (volumetric flow rate $1: 1$ ) to achieve an oxygen level of $10.5 \%$. Each cycle started with a bolus injection of microbubbles. Immediately after the injection, 500 PA frames were acquired and saved under normoxia condition, which took around 80 seconds. The gas supply to the vaporizer was then switched from normoxia condition to hypoxia condition. PA data was acquired 30 seconds after the hypoxia started, allowing the blood 
oxygenation level to stabilize under hypoxia condition. Each hypoxia session last 1.5 minutes, after which the rat was allowed to recover with normoxia condition for 10 minutes. The hypoxia-normoxia switching was repeated 3 times.

\section{Image reconstruction based on dual speed of sound}

It is critical to correctly reconstructed the scattered PA signals from microbubbles. With the setup of our in vivo imaging system, the delay-and-sum (DAS) reconstruction based on single speed of sound was not accurate. We assumed the sound speed distribution in rat tissues was homogenous, but the difference between the speed of sound in the coupling medium and rat tissue was not negligible. Therefore, dual speed of sound reconstruction was used for our in vivo system. In order to perform dual speed of sound reconstruction, we needed the information of the speed of sounds in the coupling medium and the rat tissue, as well as the location of the imaging window.

We first measured the sound speed in the coupling medium under in vivo experiment condition. Three single hair strands were placed orthogonal to the imaging plane and directly on top of the imaging window. The hair stands were imaged, and the acquired data was reconstructed by sweeping the sound speed from $1250 \mathrm{~m} / \mathrm{s}$ to $1500 \mathrm{~m} / \mathrm{s}$. The best image convergence of three hair targets indicated that the coupling medium had a sound speed of $1330 \mathrm{~m} / \mathrm{s}$.

We then determined the imaging window location. As our acoustically-transparent imaging window had negligible optical absorption at $1064 \mathrm{~nm}$ but strong absorption at $532 \mathrm{~nm}$, we used $532 \mathrm{~nm}$ to image the window. After completion of rat liver imaging, we kept the transducer at the same location and removed the rat. The illumination was switched from $1064 \mathrm{~nm}$ to $532 \mathrm{~nm}$ and the acquired PA data of the imaging window was reconstructed with the sound speed calibrated from the previous step to provide the actual location of the window. As the temperature of the rat was $37^{\circ} \mathrm{C}$, we assumed the sound speed in rat tissues was $1540 \mathrm{~m} / \mathrm{s}$. With the location of the imaging window, the dual speed of sound image reconstruction was performed.

\section{Motion detection and data stabilization}


Due to the limited pulse repetition rate of the laser, the acquired in vivo datasets were susceptible to breathing motion. Due to rat's breathing, the tissues were periodically moving in and out of the imaging plane (Supplementary Video 9). To extract the stable frames, we first picked a reference frame and calculated the normalized $2 \mathrm{D}$ crosscorrelation between the reference frame and all the other frames in the acquired dataset. The normalized cross-correlation reflects the breathing pattern of the rat, and by setting a threshold, the moving frames can be identified and removed.

\section{Oxygen saturation of hemoglobin $\left(\mathrm{sO}_{2}\right)$}

We define the $\mathrm{sO}_{2}$ at location $(\mathrm{x}, \mathrm{y})$ as [61]:

$s O_{2}(x, y)=\frac{C_{\mathrm{HbO}_{2}}(x, y)}{C_{\mathrm{HbO}_{2}}(x, y)+C_{\mathrm{HbR}}(x, y)} \times 100 \%$.

Where $C_{\mathrm{HbO}_{2}}$ and $C_{\mathrm{HbR}}$ are molar concentrations of oxygenated $\left(\mathrm{HbO}_{2}\right)$ and deoxygenated $(\mathrm{HbR})$ hemoglobin respectively. We used the linear spectral unmixing method, which states that $P\left(\lambda_{i}, x, y\right)$, the reconstructed PA image at wavelength $\lambda_{i}$ at $(x$, y) [60],

$P\left(\lambda_{i}, x, y\right)=\Phi(r, \lambda)\left(\varepsilon_{H b R}\left(\lambda_{i}\right) C_{H b R}(x, y)+\varepsilon_{H_{b O}}\left(\lambda_{i}\right) C_{H b O_{2}}(x, y)\right)$.

Here $\Phi(\lambda)$ is local optical fluence. $\varepsilon_{\mathrm{HbR}}\left(\lambda_{i}\right)$ and $\varepsilon_{\mathrm{HbO}_{2}}\left(\lambda_{i}\right)$ are the known molar extinction coefficients $\left(\mathrm{cm}^{-1} \mathrm{M}^{-1}\right)$ of $\mathrm{HbR}$ and $\mathrm{HbO}_{2}$ at wavelength $\lambda_{\mathrm{i}}$, respectively. We used an optical energy of $15 \mathrm{~mJ} /$ pulse for $532 \mathrm{~nm}$ and $90 \mathrm{~mJ} /$ pulse for $1064 \mathrm{~nm}$. After correcting for optical fluence, $C_{H b R}(x, y)$ and $C_{H b R}(x, y)$ can be estimated by solving the following linear equations:

$$
\left[\begin{array}{l}
C_{H b}(x, y) \\
C_{H b O_{2}}(x, y)
\end{array}\right]=\left(\varepsilon^{T} \varepsilon\right)^{-1} \varepsilon^{T} P .(7)
$$

where $P$ is the SVD filtered PA images at 532 and $1064 \mathrm{~nm}, \varepsilon$ is the molar extinction coefficient matrix as

$$
P=\left[\begin{array}{l}
P\left(\lambda_{1}, x, y\right) \\
P\left(\lambda_{2}, x, y\right)
\end{array}\right]
$$




$$
\varepsilon=\left[\begin{array}{ll}
\varepsilon_{H b R}\left(\lambda_{1}\right) & \varepsilon_{H_{b O}}\left(\lambda_{1}\right) \\
\varepsilon_{H b R}\left(\lambda_{2}\right) & \varepsilon_{H^{\prime} O_{2}}\left(\lambda_{2}\right)
\end{array}\right]
$$




\section{Reference}

[1] J. K. Mueller et al., "Simultaneous transcranial magnetic stimulation and singleneuron recording in alert non-human primates," Nat. Neurosci., vol. 17, no. 8, Art. no. 8, Aug. 2014, doi: 10.1038/nn.3751.

[2] A. Taruttis, A. C. Timmermans, P. C. Wouters, M. Kacprowicz, G. M. van Dam, and V. Ntziachristos, "Optoacoustic Imaging of Human Vasculature: Feasibility by Using a Handheld Probe," Radiology, vol. 281, no. 1, pp. 256-263, Oct. 2016, doi: 10.1148/radiol.2016152160.

[3] N. Nyayapathi and J. Xia, "Photoacoustic imaging of breast cancer: a mini review of system design and image features," J. Biomed. Opt., vol. 24, no. 12, p. 121911, Dec. 2019, doi: 10.1117/1.JBO.24.12.121911.

[4] L. Lin et al., "Single-breath-hold photoacoustic computed tomography of the breast," Nat. Commun., vol. 9, no. 1, p. 2352, Jun. 2018, doi: 10.1038/s41467-01804576-z.

[5] S. Manohar and M. Dantuma, "Current and future trends in photoacoustic breast imaging," Photoacoustics, vol. 16, p. 100134, Dec. 2019, doi: 10.1016/j.pacs.2019.04.004.

[6] M. Heijblom et al., "Visualizing breast cancer using the Twente photoacoustic mammoscope: What do we learn from twelve new patient measurements?," Opt. Express, vol. 20, no. 11, pp. 11582-11597, May 2012, doi: 10.1364/OE.20.011582.

[7] M. Pramanik, G. Ku, C. Li, and L. V. Wang, "Design and evaluation of a novel breast cancer detection system combining both thermoacoustic (TA) and photoacoustic (PA) tomography," Med. Phys., vol. 35, no. 6Part1, pp. 2218-2223, 2008, doi: 10.1118/1.2911157.

[8] G. Diot et al., "Multispectral Optoacoustic Tomography (MSOT) of Human Breast Cancer," Clin. Cancer Res., vol. 23, no. 22, pp. 6912-6922, Nov. 2017, doi: 10.1158/1078-0432.CCR-16-3200.

[9] E. Liapis, A. Karlas, U. Klemm, and V. Ntziachristos, "Chemotherapeutic effects on breast tumor hemodynamics revealed by eigenspectra multispectral optoacoustic tomography (eMSOT)," Theranostics, vol. 11, no. 16, pp. 7813-7828, 2021, doi: 10.7150/thno.56173.

[10] G. Balasundaram et al., "Molecular photoacoustic imaging of breast cancer using an actively targeted conjugated polymer," Int. J. Nanomedicine, vol. 10, no. 1, pp. 387397, Jan. 2015, doi: 10.2147/IJN.S73558.

[11] X. L. Deán-Ben and D. Razansky, "Optoacoustic imaging of the skin," Exp. Dermatol., vol. 30, no. 11, pp. 1598-1609, 2021, doi: 10.1111/exd.14386. 
[12] Z. Chen et al., "Non-invasive multimodal optical coherence and photoacoustic tomography for human skin imaging," Sci. Rep., vol. 7, no. 1, p. 17975, Dec. 2017, doi: 10.1038/s41598-017-18331-9.

[13] K. Kratkiewicz et al., "Photoacoustic/Ultrasound/Optical Coherence Tomography Evaluation of Melanoma Lesion and Healthy Skin in a Swine Model," Sensors, vol. 19, no. 12, p. 2815, Jun. 2019, doi: 10.3390/s19122815.

[14] K. S. Valluru, K. E. Wilson, and J. K. Willmann, "Photoacoustic Imaging in Oncology: Translational Preclinical and Early Clinical Experience," Radiology, vol. 280, no. 2, pp. 332-349, Jul. 2016, doi: 10.1148/radiol.16151414.

[15] S. Na, L. V. Wang, and L. V. Wang, "Photoacoustic computed tomography for functional human brain imaging [Invited]," Biomed. Opt. Express, vol. 12, no. 7, pp. 4056-4083, Jul. 2021, doi: 10.1364/BOE.423707.

[16] S. Gottschalk et al., "Rapid volumetric optoacoustic imaging of neural dynamics across the mouse brain," Nat. Biomed. Eng., p. 1, Mar. 2019, doi: 10.1038/s41551-0190372-9.

[17] D. Razansky, J. Klohs, and R. Ni, "Multi-scale optoacoustic molecular imaging of brain diseases," Eur. J. Nucl. Med. Mol. Imaging, vol. 48, no. 13, pp. 4152-4170, Dec. 2021, doi: 10.1007/s00259-021-05207-4.

[18] X. L. Deán-Ben et al., "Functional optoacoustic neuro-tomography for scalable whole-brain monitoring of calcium indicators," Light Sci. Appl., vol. 5, no. 12, Art. no. 12, Dec. 2016, doi: 10.1038/lsa.2016.201.

[19] X. Luís Deán-Ben and D. Razansky, "Adding fifth dimension to optoacoustic imaging: volumetric time-resolved spectrally enriched tomography," Light Sci. Appl., vol. 3, no. 1, pp. e137-e137, Jan. 2014, doi: 10.1038/lsa.2014.18.

[20] S. V. Ovsepian, I. Olefir, G. Westmeyer, D. Razansky, and V. Ntziachristos, "Pushing the Boundaries of Neuroimaging with Optoacoustics," Neuron, vol. 96, no. 5, pp. 966-988, Dec. 2017, doi: 10.1016/j.neuron.2017.10.022.

[21] A. B. E. Attia et al., "Multispectral optoacoustic and MRI coregistration for molecular imaging of orthotopic model of human glioblastoma," J. Biophotonics, vol. 9, no. 7, pp. 701-708, 2016, doi: 10.1002/jbio.201500321.

[22] I. Olefir, E. Merčep, N. C. Burton, S. V. Ovsepian, and V. Ntziachristos, "Hybrid multispectral optoacoustic and ultrasound tomography for morphological and physiological brain imaging," J. Biomed. Opt., vol. 21, no. 8, p. 086005, Aug. 2016, doi: 10.1117/1.JBO.21.8.086005.

[23] X. L. Deán-Ben, S. Gottschalk, G. Sela, S. Shoham, and D. Razansky, "Functional optoacoustic neuro-tomography of calcium fluxes in adult zebrafish brain in vivo," Opt. Lett., vol. 42, no. 5, pp. 959-962, Mar. 2017, doi: 10.1364/OL.42.000959. 
[24] P. Beard, "Biomedical photoacoustic imaging," Interface Focus, Aug. 2011, Accessed: Sep. 20, 2019. [Online]. Available:

https://royalsocietypublishing.org/doi/abs/10.1098/rsfs.2011.0028

[25] J. Xia, J. Yao, and L. V. Wang, "Photoacoustic tomography: principles and advances," Electromagn. Waves Camb. Mass, vol. 147, pp. 1-22, 2014.

[26] X. L. Deán-Ben, L. Ding, and D. Razansky, "Dynamic particle enhancement in limited-view optoacoustic tomography," Opt. Lett., vol. 42, no. 4, pp. 827-830, Feb. 2017, doi: 10.1364/OL.42.000827.

[27] X. L. Deán-Ben and D. Razansky, "On the link between the speckle free nature of optoacoustics and visibility of structures in limited-view tomography," Photoacoustics, vol. 4, no. 4, pp. 133-140, Dec. 2016, doi: 10.1016/j.pacs.2016.10.001.

[28] X. Luís Dean-Ben and D. Razansky, "Localization optoacoustic tomography," Light Sci. Appl., vol. 7, no. 4, pp. 18004-18004, Apr. 2018, doi: 10.1038/lsa.2018.4.

[29] G. Paltauf, R. Nuster, M. Haltmeier, and P. Burgholzer, "Experimental evaluation of reconstruction algorithms for limited view photoacoustic tomography with line detectors," Inverse Problems, vol. 23, no. 6, pp. S81-S94, Nov. 2007, doi: 10.1088/0266-5611/23/6/S07.

[30] P. Zhang et al., "High-resolution deep functional imaging of the whole mouse brain by photoacoustic computed tomography in vivo," J. Biophotonics, vol. 11, no. 1, p. e201700024, Jan. 2018, doi: 10.1002/jbio.201700024.

[31] H.-P. Brecht, R. Su, M. Fronheiser, S. A. Ermilov, A. Conjusteau, and A. A. Oraevsky, "Whole-body three-dimensional optoacoustic tomography system for small animals," J. Biomed. Opt., vol. 14, no. 6, p. 064007, 2009, doi: 10.1117/1.3259361.

[32] Y. Zhang and L. Wang, "Video-Rate Ring-Array Ultrasound and Photoacoustic Tomography," IEEE Trans. Med. Imaging, vol. 39, no. 12, pp. 4369-4375, Dec. 2020, doi: 10.1109/TMI.2020.3017815.

[33] M. Nishiyama, T. Namita, K. Kondo, M. Yamakawa, and T. Shiina, "Ring-array photoacoustic tomography for imaging human finger vasculature," J. Biomed. Opt., vol. 24, no. 9, p. 096005, Sep. 2019, doi: 10.1117/1.JBO.24.9.096005.

[34] P. Zhang, L. Li, L. Lin, J. Shi, and L. V. Wang, "In vivo superresolution photoacoustic computed tomography by localization of single dyed droplets," Light Sci. Appl., vol. 8, no. 1, p. 36, Apr. 2019, doi: 10.1038/s41377-019-0147-9.

[35] N. Davoudi, X. L. Deán-Ben, and D. Razansky, "Deep learning optoacoustic tomography with sparse data," Nat. Mach. Intell., vol. 1, no. 10, pp. 453-460, Oct. 2019, doi: 10.1038/s42256-019-0095-3. 
[36] J. Gröhl, M. Schellenberg, K. Dreher, and L. Maier-Hein, "Deep learning for biomedical photoacoustic imaging: A review," Photoacoustics, vol. 22, p. 100241, Jun. 2021, doi: 10.1016/j.pacs.2021.100241.

[37] S. Guan, A. A. Khan, S. Sikdar, and P. V. Chitnis, "Limited-View and Sparse Photoacoustic Tomography for Neuroimaging with Deep Learning," Sci. Rep., vol. 10, no. 1, p. 8510, May 2020, doi: 10.1038/s41598-020-65235-2.

[38] S. Antholzer, M. Haltmeier, and J. Schwab, "Deep learning for photoacoustic tomography from sparse data," Inverse Probl. Sci. Eng., vol. 27, no. 7, pp. 987-1005, Jul. 2019, doi: 10.1080/17415977.2018.1518444.

[39] M. Kim, G.-S. Jeng, I. Pelivanov, and M. O'Donnell, "Deep-Learning Image Reconstruction for Real-Time Photoacoustic System," IEEE Trans. Med. Imaging, vol. 39, no. 11, pp. 3379-3390, Nov. 2020, doi: 10.1109/TMI.2020.2993835.

[40] R. Manwar, X. Li, S. Mahmoodkalayeh, E. Asano, D. Zhu, and K. Avanaki, "Deep learning protocol for improved photoacoustic brain imaging," J. Biophotonics, vol. 13, no. 10, p. e202000212, 2020, doi: 10.1002/jbio.202000212.

[41] T. Vu, M. Li, H. Humayun, Y. Zhou, and J. Yao, "A generative adversarial network for artifact removal in photoacoustic computed tomography with a linear-array transducer," Exp. Biol. Med., vol. 245, no. 7, pp. 597-605, Apr. 2020, doi: $10.1177 / 1535370220914285$.

[42] A. DiSpirito, T. Vu, M. Pramanik, and J. Yao, "Sounding out the hidden data: A concise review of deep learning in photoacoustic imaging," Exp. Biol. Med. Maywood NJ, vol. 246, no. 12, pp. 1355-1367, Jun. 2021, doi: 10.1177/15353702211000310.

[43] S. R. Wilson and P. N. Burns, "Microbubble-enhanced US in Body Imaging: What Role?," Radiology, vol. 257, no. 1, pp. 24-39, Oct. 2010, doi: 10.1148/radiol.10091210.

[44] P. Song et al., "Improved Super-Resolution Ultrasound Microvessel Imaging With Spatiotemporal Nonlocal Means Filtering and Bipartite Graph-Based Microbubble Tracking," IEEE Trans. Ultrason. Ferroelectr. Freq. Control, vol. 65, no. 2, pp. 149-167, Feb. 2018, doi: 10.1109/TUFFC.2017.2778941.

[45] K. Wei, E. Le, J.-P. Bin, M. Coggins, J. Thorpe, and S. Kaul, "Quantification of renal blood flow with contrast-enhanced ultrasound," J. Am. Coll. Cardiol., vol. 37, no. 4, pp. 1135-1140, Mar. 2001, doi: 10.1016/S0735-1097(00)01210-9.

[46] E. C. Unger, T. Porter, W. Culp, R. Labell, T. Matsunaga, and R. Zutshi, "Therapeutic applications of lipid-coated microbubbles," Adv. Drug Deliv. Rev., vol. 56, no. 9, pp. 1291-1314, May 2004, doi: 10.1016/j.addr.2003.12.006.

[47] C. F. Dietrich, M. A. Averkiou, J.-M. Correas, N. Lassau, E. Leen, and F. Piscaglia, "An EFSUMB Introduction into Dynamic Contrast-Enhanced Ultrasound 
(DCE-US) for Quantification of Tumour Perfusion," Ultraschall Med. - Eur. J. Ultrasound, vol. 33, no. 04, pp. 344-351, Aug. 2012, doi: 10.1055/s-0032-1313026.

[48] C. Sonne et al., "Differences in definity and optison microbubble destruction rates at a similar mechanical index with different real-time perfusion systems," J. Am. Soc. Echocardiogr., vol. 16, no. 11, pp. 1178-1185, Nov. 2003, doi: 10.1067/j.echo.2003.07.001.

[49] S. Sirsi and M. Borden, "Microbubble Compositions, Properties and Biomedical Applications," Bubble Sci. Eng. Technol., vol. 1, no. 1-2, p. 3, Nov. 2009, doi: 10.1179/175889709X446507.

[50] V. S. Lucas, R. S. Burk, S. Creehan, and M. J. Grap, "Utility of High-Frequency Ultrasound: Moving Beyond the Surface to Detect Changes in Skin Integrity," Plast. Surg. Nurs. Off. J. Am. Soc. Plast. Reconstr. Surg. Nurses, vol. 34, no. 1, p. 34, Mar. 2014, doi: 10.1097/PSN.0000000000000031.

[51] B. E. Treeby and B. T. Cox, "k-Wave: MATLAB toolbox for the simulation and reconstruction of photoacoustic wave fields," J. Biomed. Opt., vol. 15, no. 2, p. 021314, Mar. 2010, doi: 10.1117/1.3360308.

[52] G. Pinton, "Three dimensional full-wave nonlinear acoustic simulations of ultrasound imaging and therapy in the entire human body," in 2012 IEEE International Ultrasonics Symposium, Oct. 2012, pp. 142-145. doi: 10.1109/ULTSYM.2012.0035.

[53] M.-X. Tang and R. J. Eckersley, "Frequency and pressure dependent attenuation and scattering by microbubbles," Ultrasound Med. Biol., vol. 33, no. 1, pp. 164-168, Jan. 2007, doi: 10.1016/j.ultrasmedbio.2006.07.031.

[54] B. E. Treeby, E. Z. Zhang, and B. T. Cox, "Photoacoustic tomography in absorbing acoustic media using time reversal," Inverse Probl., vol. 26, no. 11, p. 115003, Sep. 2010, doi: 10.1088/0266-5611/26/11/115003.

[55] A. Rodriguez-Molares et al., "The generalized contrast-to-noise ratio: a formal definition for lesion detectability," IEEE Trans. Ultrason. Ferroelectr. Freq. Control, vol. 67, no. 4, pp. 745-759, Apr. 2020, doi: 10.1109/TUFFC.2019.2956855.

[56] R. Ma, A. Taruttis, V. Ntziachristos, and D. Razansky, "Multispectral optoacoustic tomography (MSOT) scanner for whole-body small animal imaging," Opt. Express, vol. 17, no. 24, pp. 21414-21426, Nov. 2009, doi: 10.1364/OE.17.021414.

[57] A. Bjørnerud, K. Briley-Sæbø, L. O. Johansson, and K. E. Kellar, "Effect of NC100150 injection on the $1 \mathrm{H}$ NMR linewidth of human whole blood ex vivo: Dependency on blood oxygen tension," Magn. Reson. Med., vol. 44, no. 5, pp. 803807, Nov. 2000, doi: 10.1002/1522-2594(200011)44:5<803::AID-MRM19>3.0.CO;2-K. 
[58] B. Seaton and B. B. Lloyd, "The effects of $\mathrm{pH}$ on the equilibrium constants of various models for the haemoglobin-oxygen equilibrium in vitro," Respir. Physiol., vol. 20, no. 2, pp. 209-230, Mar. 1974, doi: 10.1016/0034-5687(74)90108-X.

[59] M. Li, Y. Tang, and J. Yao, "Photoacoustic tomography of blood oxygenation: A mini review," Photoacoustics, vol. 10, pp. 65-73, Jun. 2018, doi:

10.1016/j.pacs.2018.05.001.

[60] M.-L. Li et al., "Simultaneous Molecular and Hypoxia Imaging of Brain Tumors In Vivo Using Spectroscopic Photoacoustic Tomography," Proc. IEEE, vol. 96, no. 3, pp. 481-489, Mar. 2008, doi: 10.1109/JPROC.2007.913515.

[61] L. J, E. C, D. D, and B. P, "In vitro measurements of absolute blood oxygen saturation using pulsed near-infrared photoacoustic spectroscopy: accuracy and resolution," Phys. Med. Biol., vol. 50, no. 18, Sep. 2005, doi: 10.1088/00319155/50/18/011.

[62] A. Mustari et al., "Agarose-based Tissue Mimicking Optical Phantoms for Diffuse Reflectance Spectroscopy," J. Vis. Exp. JoVE, no. 138, p. 57578, Aug. 2018, doi: $10.3791 / 57578$.

[63] T. Chaigne, B. Arnal, S. Vilov, E. Bossy, and O. Katz, "Super-resolution photoacoustic imaging via flow-induced absorption fluctuations," Optica, vol. 4, no. 11, pp. 1397-1404, Nov. 2017, doi: 10.1364/OPTICA.4.001397.

[64] F. Xu, P. Liu, J. M. Pascual, G. Xiao, and H. Lu, "Effect of hypoxia and hyperoxia on cerebral blood flow, blood oxygenation, and oxidative metabolism," J. Cereb. Blood Flow Metab. Off. J. Int. Soc. Cereb. Blood Flow Metab., vol. 32, no. 10, pp. 1909-1918, Oct. 2012, doi: 10.1038/jcbfm.2012.93.

[65] C. Errico et al., "Ultrafast ultrasound localization microscopy for deep superresolution vascular imaging," Nature, vol. 527, no. 7579, pp. 499-502, Nov. 2015, doi: 10.1038/nature16066.

[66] G. Wissmeyer, M. A. Pleitez, A. Rosenthal, and V. Ntziachristos, "Looking at sound: optoacoustics with all-optical ultrasound detection," Light Sci. Appl., vol. 7, no. 1, p. 53, Aug. 2018, doi: 10.1038/s41377-018-0036-7.

[67] P. G. Westergaard and M. Lassen, "All-optical detection of acoustic pressure waves with applications in photoacoustic spectroscopy," Appl. Opt., vol. 55, no. 29, pp. 8266-8270, Oct. 2016, doi: 10.1364/AO.55.008266.

[68] B. Dong, C. Sun, and H. F. Zhang, "Optical Detection of Ultrasound in Photoacoustic Imaging," IEEE Trans. Biomed. Eng., vol. 64, no. 1, pp. 4-15, Jan. 2017, doi: 10.1109/TBME.2016.2605451.

[69] C. Cai, X. Wang, K. Si, J. Qian, J. Luo, and C. Ma, "Feature coupling photoacoustic computed tomography for joint reconstruction of initial pressure and 
sound speed in vivo," Biomed. Opt. Express, vol. 10, no. 7, pp. 3447-3462, Jul. 2019, doi: 10.1364/BOE.10.003447.

[70] P. Song, A. Manduca, J. D. Trzasko, and S. Chen, "Ultrasound Small Vessel Imaging With Block-Wise Adaptive Local Clutter Filtering," IEEE Trans. Med. Imaging, vol. 36, no. 1, pp. 251-262, Jan. 2017, doi: 10.1109/TMI.2016.2605819.

[71] S. P et al., "Functional Ultrasound Imaging of Spinal Cord Hemodynamic Responses to Epidural Electrical Stimulation: A Feasibility Study," Front. Neurol., vol. 10, Mar. 2019, doi: 10.3389/fneur.2019.00279.

[72] P. Song, A. Manduca, J. D. Trzasko, and S. Chen, "Ultrasound Small Vessel Imaging With Block-Wise Adaptive Local Clutter Filtering," IEEE Trans. Med. Imaging, vol. 36, no. 1, pp. 251-262, Jan. 2017, doi: 10.1109/TMI.2016.2605819.

[73] M. Emmer, A. van Wamel, D. E. Goertz, and N. de Jong, "The Onset of Microbubble Vibration," Ultrasound Med. Biol., vol. 33, no. 6, pp. 941-949, Jun. 2007, doi: 10.1016/j.ultrasmedbio.2006.11.004.

[74] A. C. H. Yu and L. Lovstakken, "Eigen-based clutter filter design for ultrasound color flow imaging: a review," IEEE Trans. Ultrason. Ferroelectr. Freq. Control, vol. 57, no. 5, pp. 1096-1111, May 2010, doi: 10.1109/TUFFC.2010.1521.

[75] L. A. Ledoux, P. J. Brands, and A. P. Hoeks, "Reduction of the clutter component in Doppler ultrasound signals based on singular value decomposition: a simulation study," Ultrason. Imaging, vol. 19, no. 1, pp. 1-18, Jan. 1997, doi: 10.1177/016173469701900101.

[76] S. Bjaerum, H. Torp, and K. Kristoffersen, "Clutter filters adapted to tissue motion in ultrasound color flow imaging," IEEE Trans. Ultrason. Ferroelectr. Freq. Control, vol. 49, no. 6, pp. 693-704, Jun. 2002, doi: 10.1109/tuffc.2002.1009328.

[77] F. Song, D. Zhang, and X. Gong, "Performance evaluation of eigendecomposition-based adaptive clutter filter for color flow imaging," Ultrasonics, vol. 44 Suppl 1, pp. e67-71, Dec. 2006, doi: 10.1016/j.ultras.2006.06.039. 


\section{Figures and captions}

a

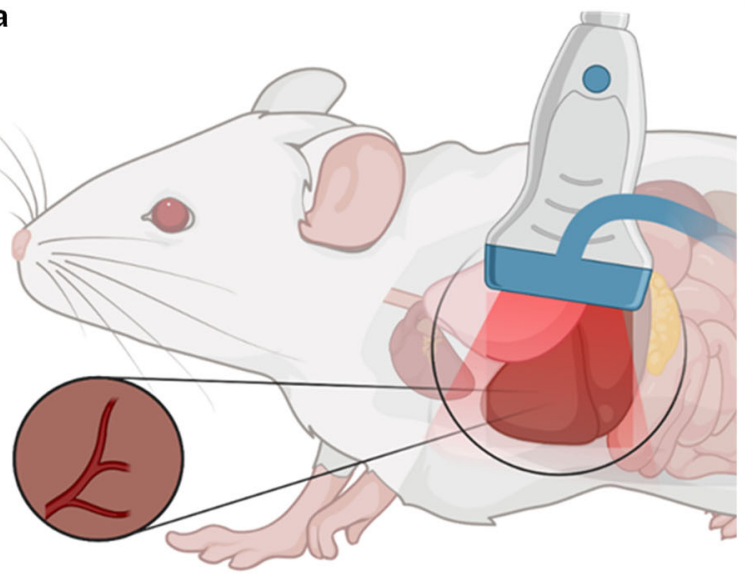

C

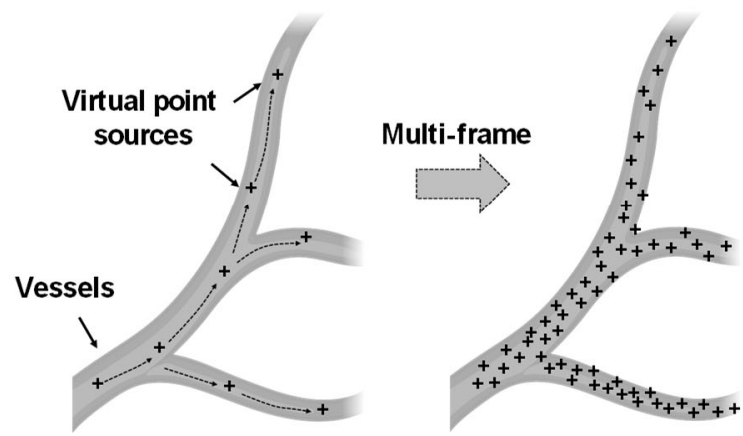

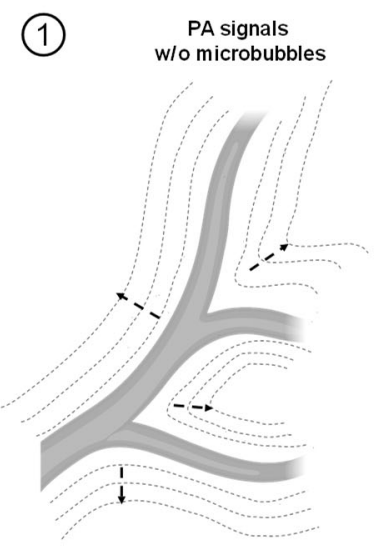

(3)

PA signals scattered
by microbubble at timepoint 2

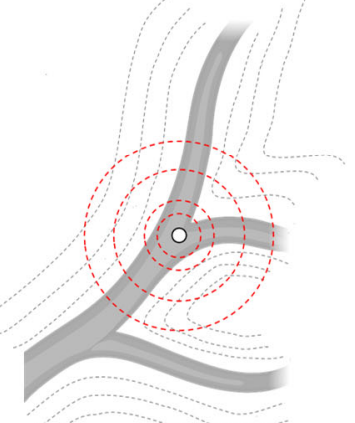

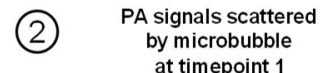
by microbubble at timepoint 1

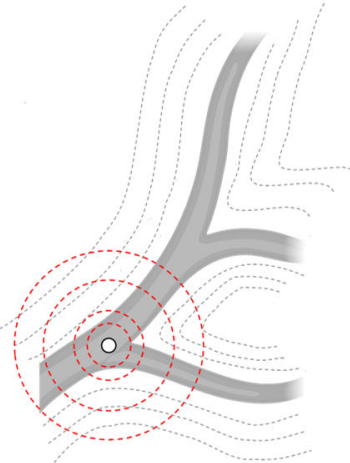

(4) PA signals after Microbubble detection

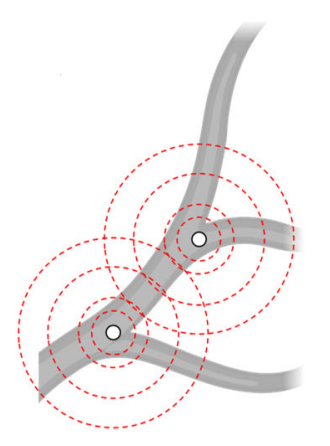

PA signals directly from blood

PA signals scattered by microbubble

Figure 1. Working principle of PAT enhanced by microbubbles as virtual point sources (VPS-PAT).

(a) Schematic of traditional photoacoustic (PA) tomography (PAT) with a linear transducer array. Pulsed laser light is delivered with a linear bifurcated fiber attached at the sides of the linear transducer array. (b) Schematics of VPS-PAT with microbubbles. (b.1) The PA waves generated by the vessels (gray dashed lines) generally travel along the vessel orientations, as indicated by the arrows. The linear array transducer (not shown) placed on top of vessels is unable to detect side travelling PA waves. (b.2-3) The spherical PA waves scattered by a flowing microbubble (red dashed circles) at two different timepoints. (b.4) By removing spatiotemporally correlated signals directly from blood, only PA signals redirected by the microbubble is preserved. (c) Scattered PA signals by the microbubbles can be reconstructed as virtual point sources. By accumulating multiple frames at different timepoints, the complete vessel structure can be reconstructed. 
a
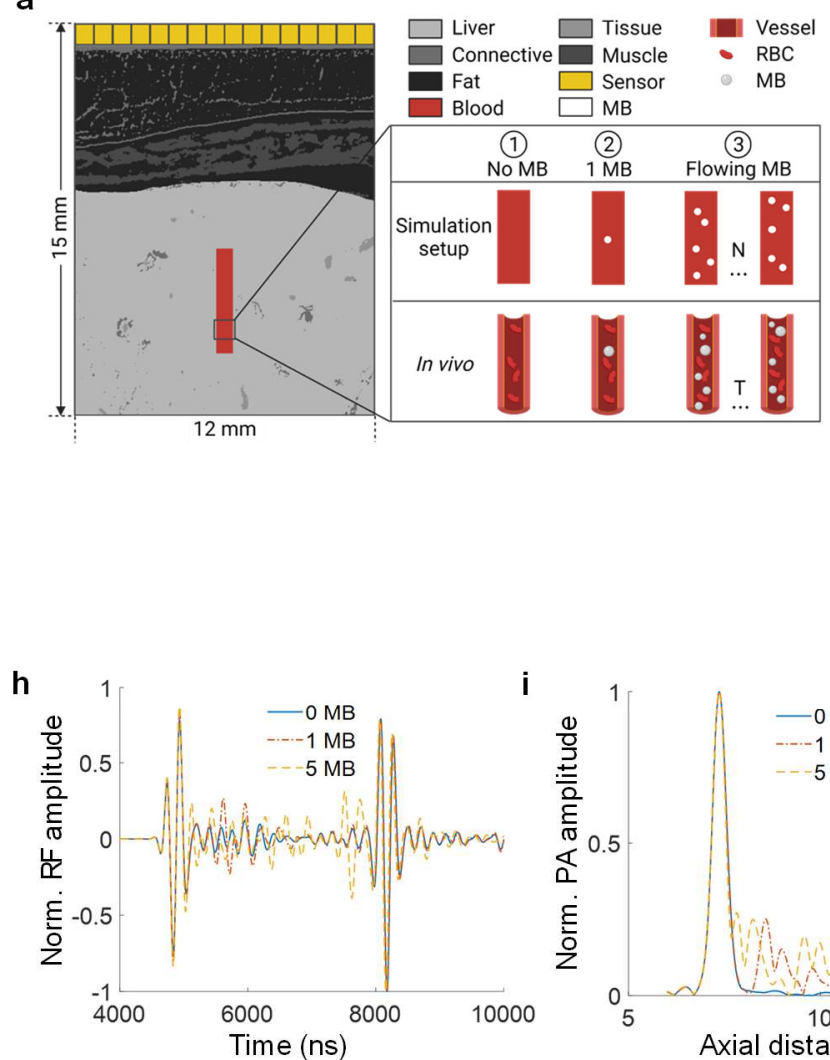

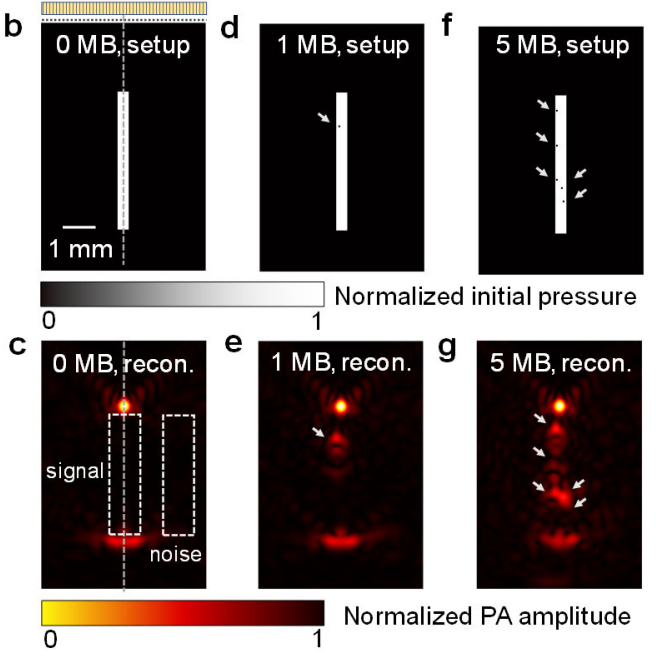

。
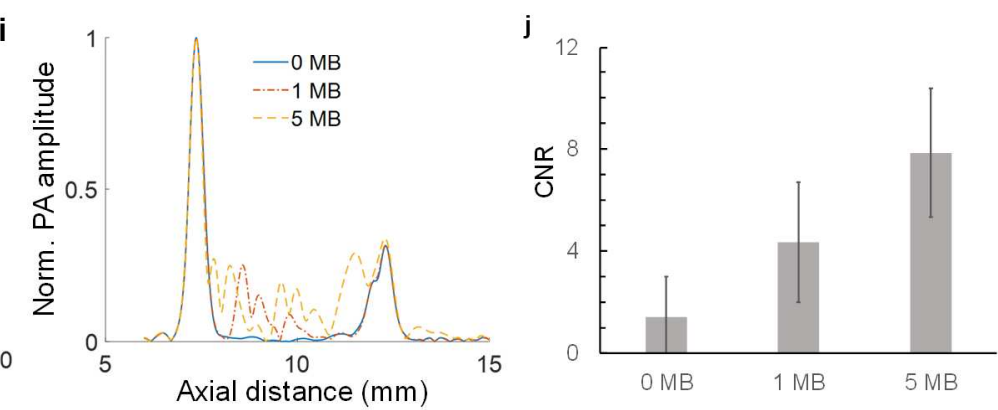

Figure 2. Simulation of microbubbles as virtual point sources in PAT. (a) The $12 \times 15 \mathrm{~mm}^{2}$ simulation region included different types of soft tissues. The linear array transducer was placed intact with skin surface, and a $0.2 \times 5 \mathrm{~mm}^{2}$ cylinder represented a vertical blood vessel. Three conditions were simulated: (1) no microbubble in the vessel, (2) a single static microbubble in the vessel at one timepoint, and (3) flowing microbubbles at $\mathrm{N}$ timepoints. (b-g) Simulation setup with 0,1 , and 5 static microbubbles placed inside the vessel respectively, and the corresponding reconstructed PA images. The microbubbles were clearly reconstructed as virtual point sources. (h) Extracted RF data along the dashed line passing through the middle of the vessel, as indicated in (b), for 0,1 , and 5 static microbubbles. (i) Extracted intensity profile from the reconstructed images along the dashed line passing through the middle of the vessel, as indicated in (c), for 0,1 , and 5 static microbubbles. (j) Comparison of CNR in reconstructed B-mode images with signal and noise regions defined by the dashed boxes in (c), for 0,1 , and 5 microbubbles respectively. 
a

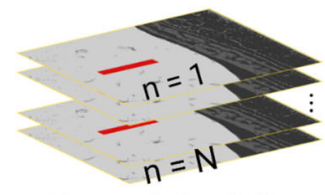

Forward simulation

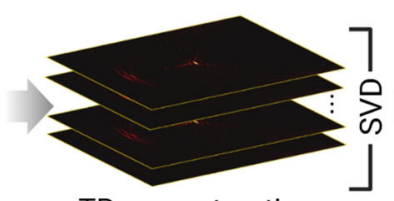

TR reconstruction

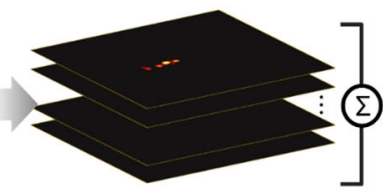

MB signal extraction

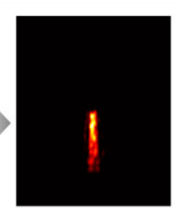

Recovered stucture

b

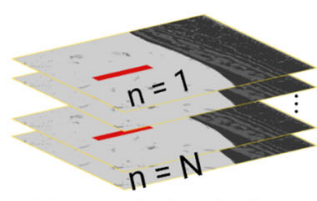

Forward simulation

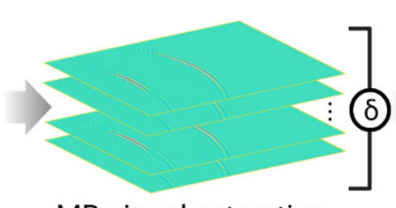

MB signal extraction

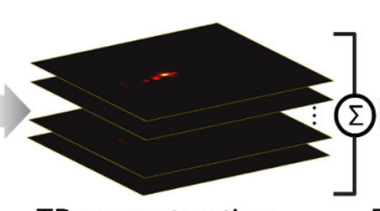

TR reconstruction

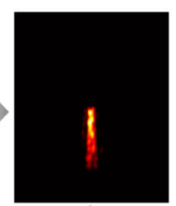

Recovered stucture
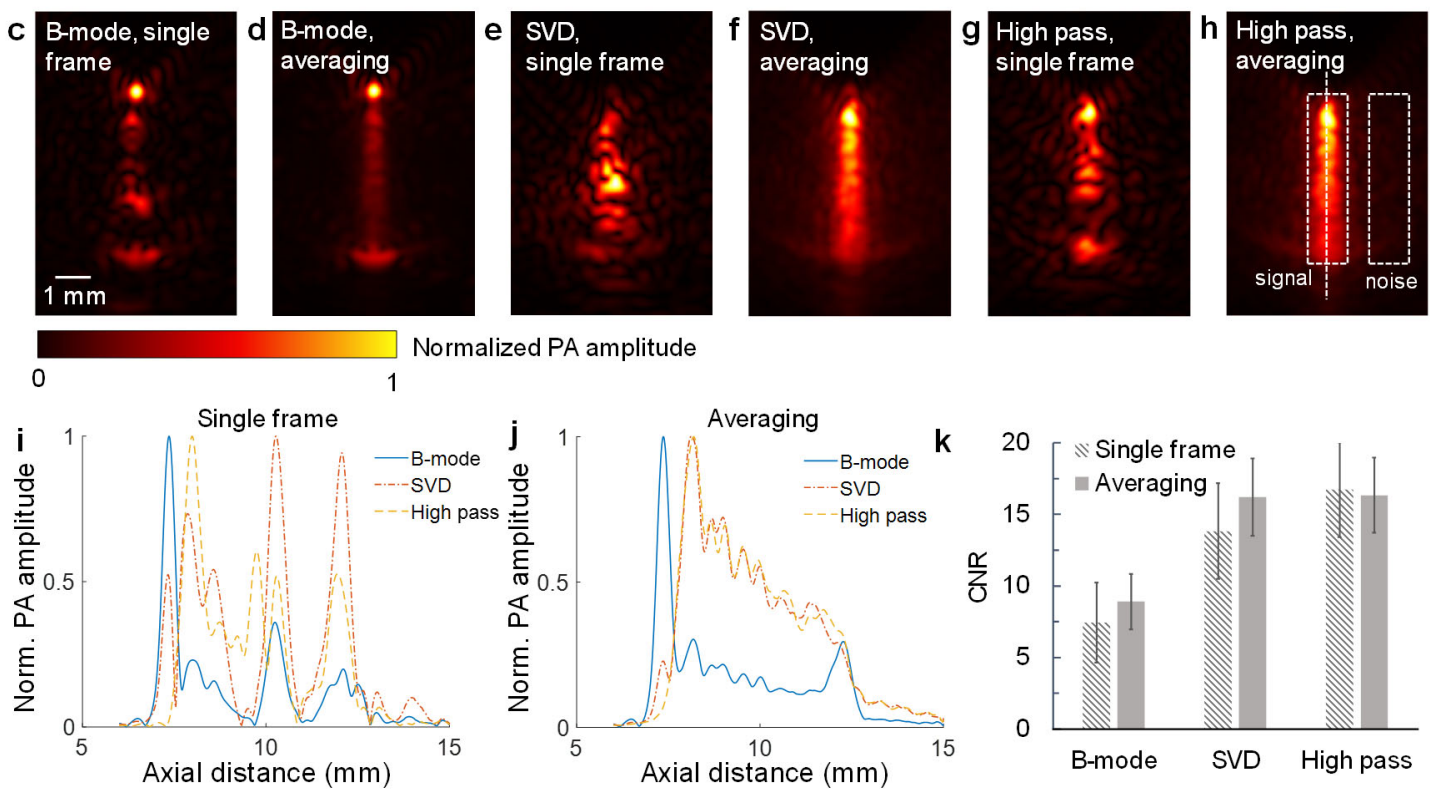

Figure 3. Data processing schematics of VPS-PAT with improved visibility of vertical structure. (a) Extracting microbubble signals with a singular value decomposition (SVD) filter on the reconstructed images. TR, time reversal. (b) Extracting microbubble signals with a high-pass filter on the received channel data. (c) Single B-mode PA image of the vessel with 5 microbubbles. (d) Averaged B-mode PA image over 20 frames. (e) Single SVD-filtered PA image of the vessel. (f) Averaged SVD-filtered image. (g) Single highpass-filtered PA image of the vessel. (h) Averaged high-pass-filtered PA image. (i-j) Extracted intensity profile along the dashed line passing through the middle of the vessel, as indicated in $(h)$, for single-frame and averaged B-mode, SVD-filtered, and high-pass-filtered images with 5 flowing microbubbles. (k) Comparison of CNR in single and averaged frames of 5 flowing microbubbles in vessel, processed with the SVD filter or high-pass filter. Signal and noise regions are defined by the dashed boxes in (h). 


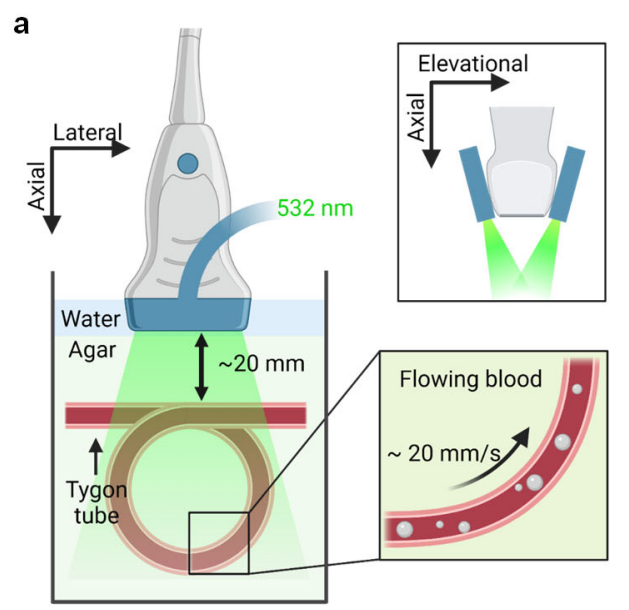

b
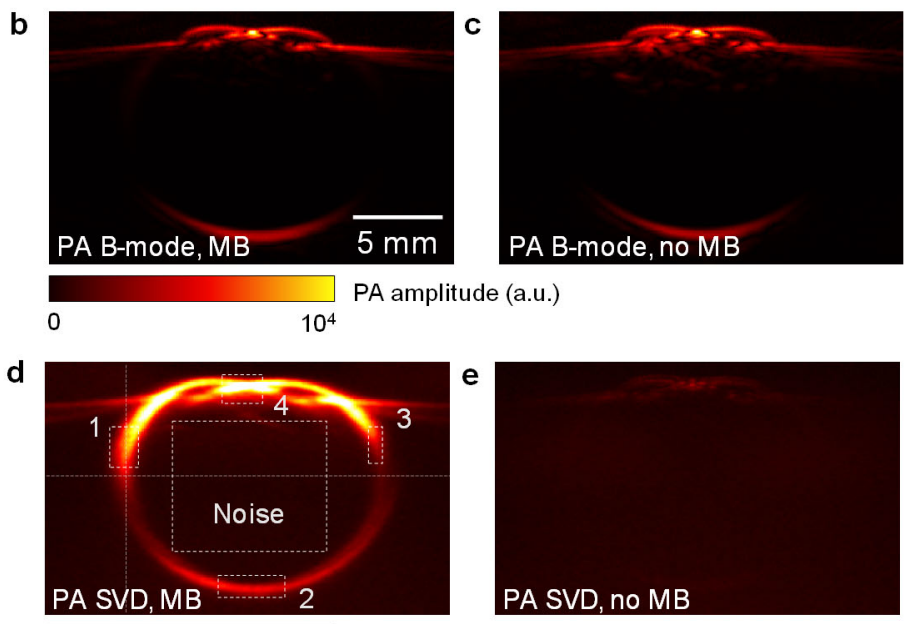

0 300

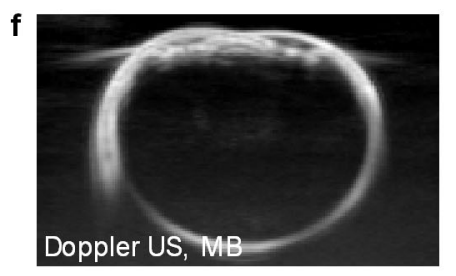

US amplitude (a.u.)

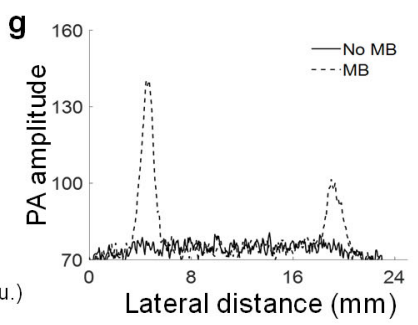

Lateral distance $(\mathrm{mm})$
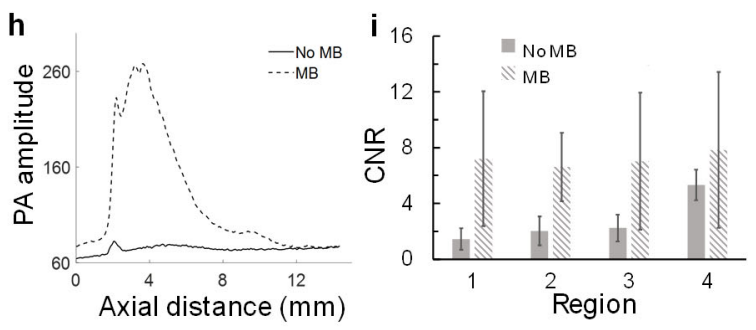

Figure 4. In vitro VPS-PAT of a knot tube with flowing blood-microbubble mixture. (a) Schematic of in vitro imaging setup. A 300- $\mu \mathrm{m}$-diameter capillary tube knot was placed $20 \mathrm{~mm}$ from the transducer. Blood-microbubble mixture was perfused with a syringe pump at a flow speed of $20 \mathrm{~mm} / \mathrm{s}$. (b-c) B-mode PA image of the tube with and without the microbubbles. (d-e) SVD filtered images of the tube with and without microbubbles. (f) Doppler US image of the tube with microbubbles. (g-h) Extracted intensity profile in the SVD filtered PA image along lateral and axial lines defined by the dashed lines in (d). (i) CNR comparison of four representative horizontal and vertical regions of the SVD-filtered images with and without microbubbles. The four regions were indicated by the dashed boxes in (d). 
a

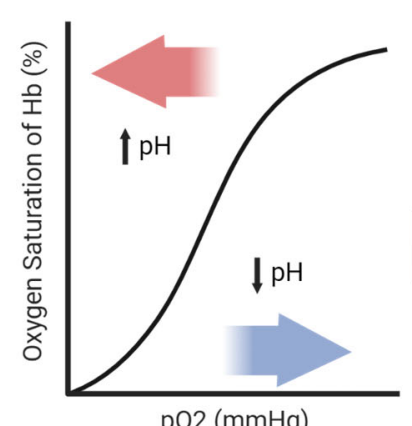

pO2 $(\mathrm{mmHg})$

C

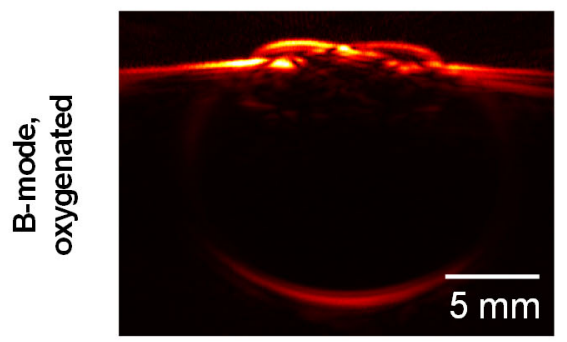

d
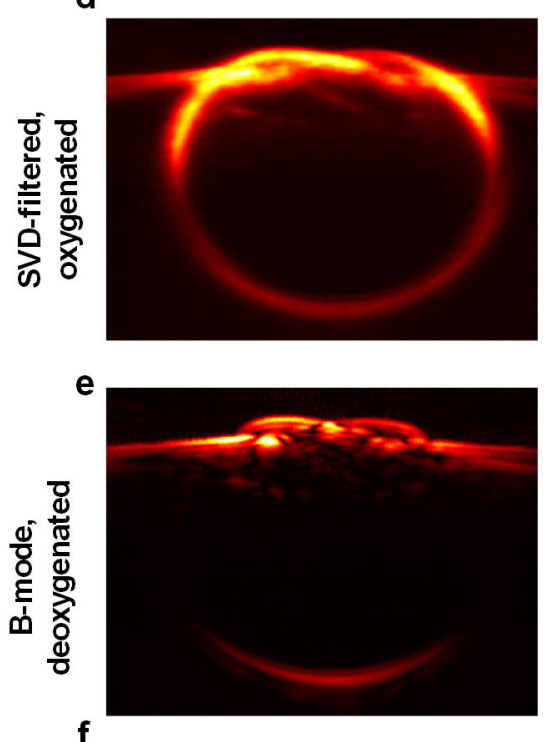

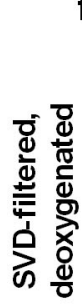

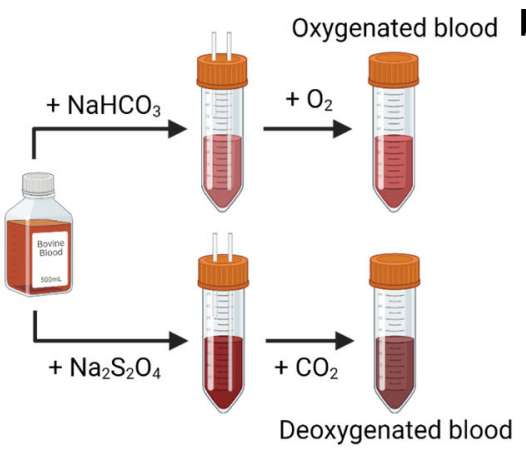

PA, $1064 \mathrm{~nm}$
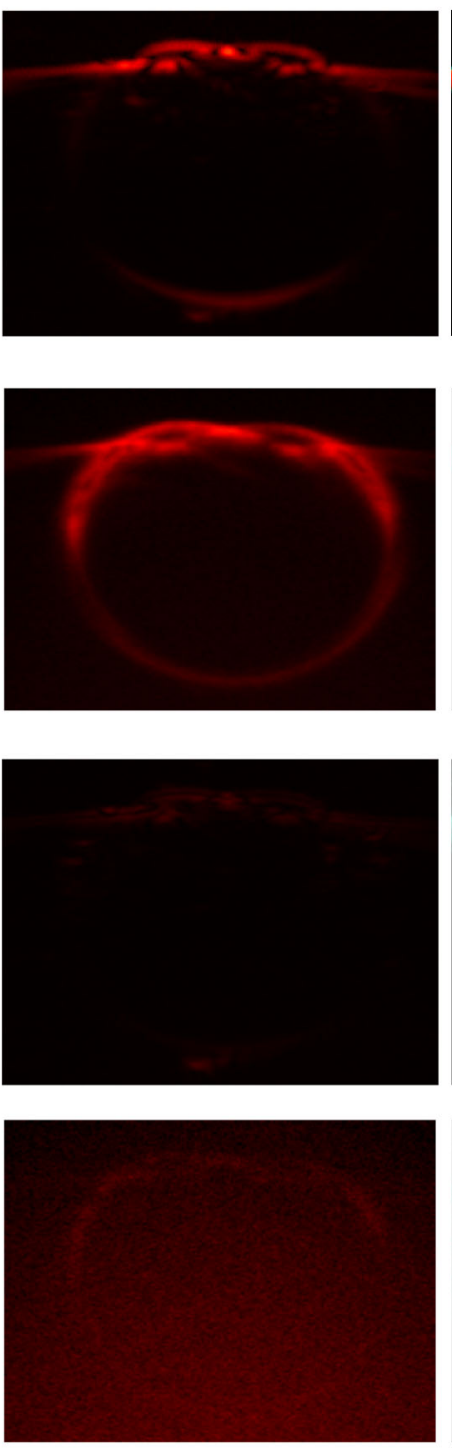

PA amplitude (a.u.)

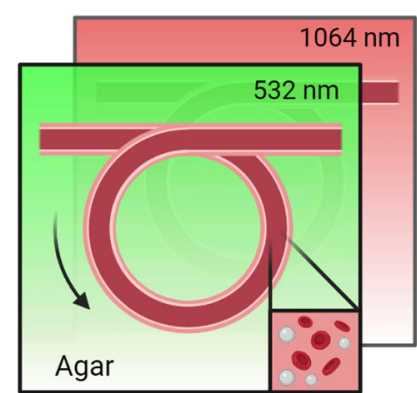

$\mathrm{sO}_{2}$
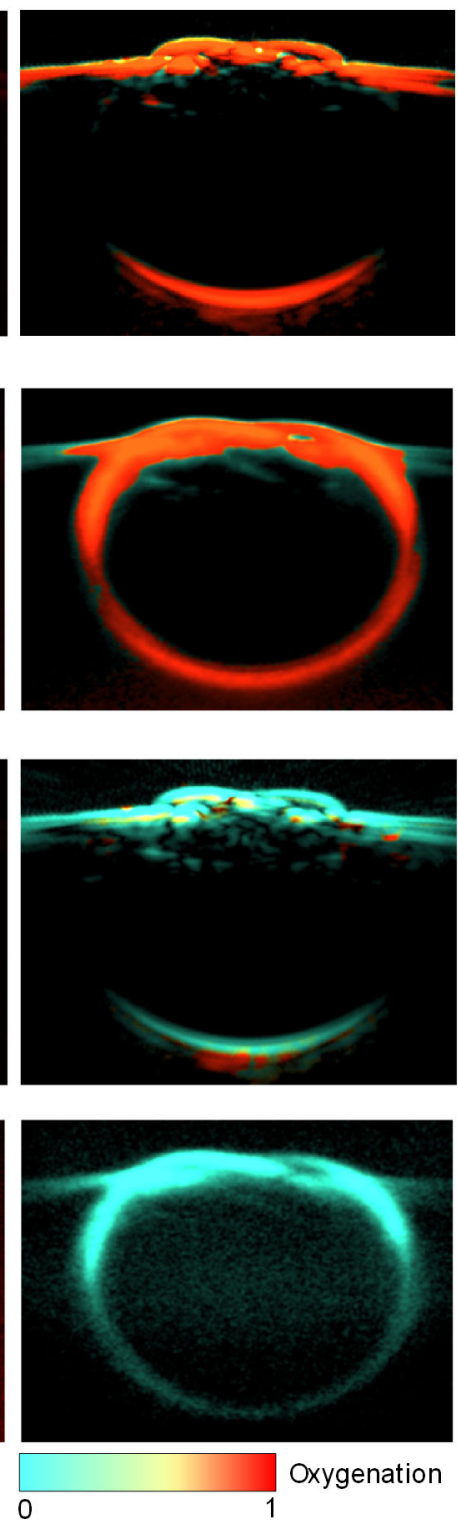

Figure 5. Multispectral functional VPS-PAT of blood oxygenation. (a) Schematics of preparing oxygenated and deoxygenated blood. (b) Schematic of imaging the blood tube with microbubbles at 532 
and $1064 \mathrm{~nm}$. (c-d) PA B-mode (c) and SVD-filtered (d) images of the tube perfused with oxygenated blood at $532 \mathrm{~nm}$ and $1064 \mathrm{~nm}$, as well as the computed $\mathrm{sO}_{2}$ image. (e-f) PA B-mode (e) and SVD-filtered (f) images of the tube perfused with deoxygenated blood at $532 \mathrm{~nm}$ and $1064 \mathrm{~nm}$, as well as the computed $\mathrm{sO}_{2}$ image. 
a

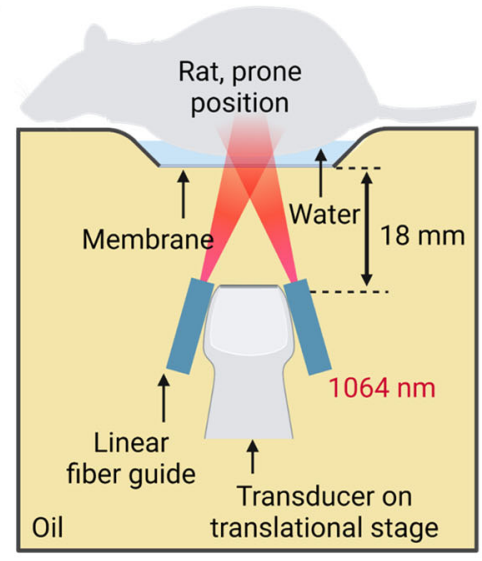

e

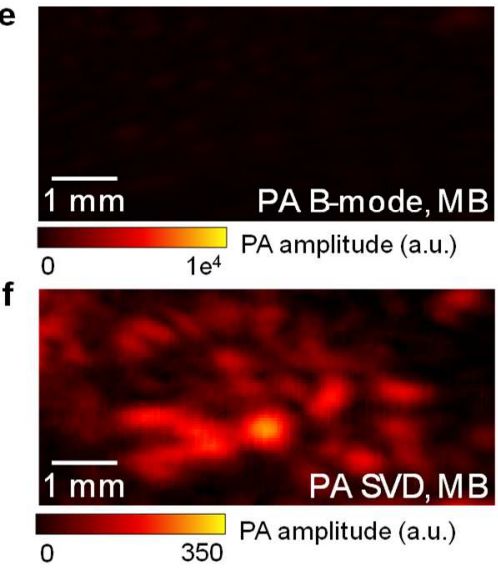

b

\section{PA B-mode, MB}

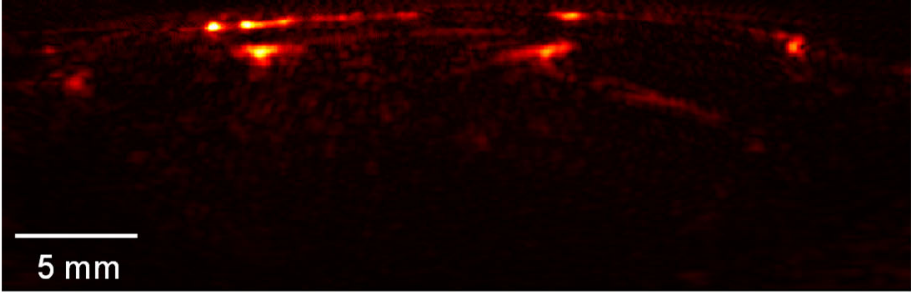

PA amplitude (a.u.)

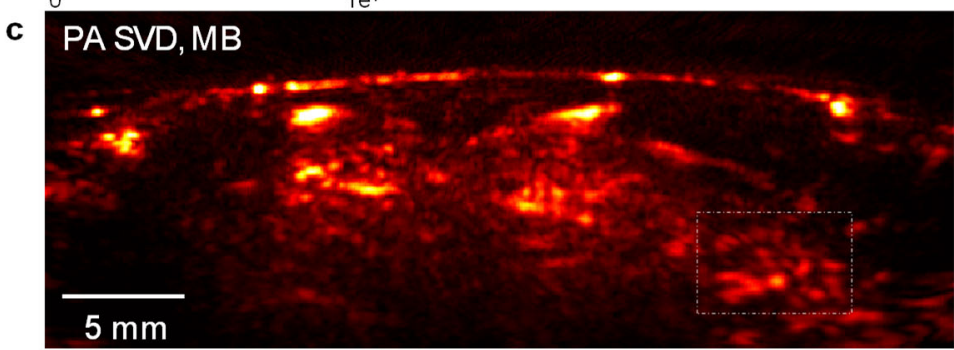

PA amplitude (a.u.)

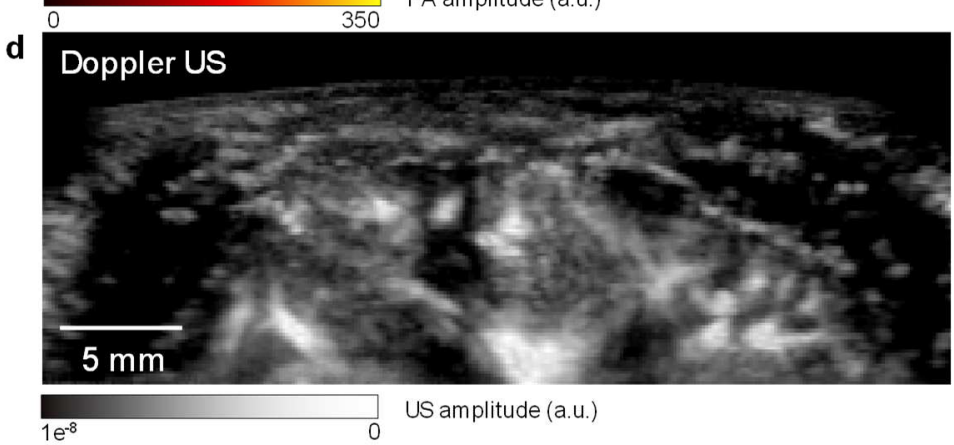

Figure 6: In vivo VPS-PAT of rat liver. (a) Schematic of in vivo imaging. Rat was placed in prone position on an acoustic-transparent membrane. The linear array transducer and the linear bifurcated fiber were immersed in oil beneath the membrane. Rat skin is around $18 \mathrm{~mm}$ from the transducer surface. (b-c) Bmode and averaged SVD-filtered PA images with microbubble injection. (d) Doppler ultrasound image of rat liver with microbubble injection. Used as a reference for anatomical structure. (e-f) Regional structure comparison between B-mode (e) and SVD-filtered (f) images, with injection. Region is indicated in (c) with dashed box. 
a

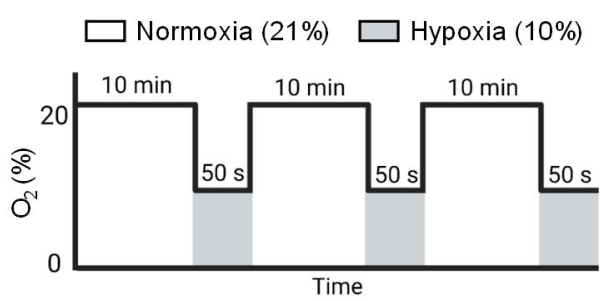

d

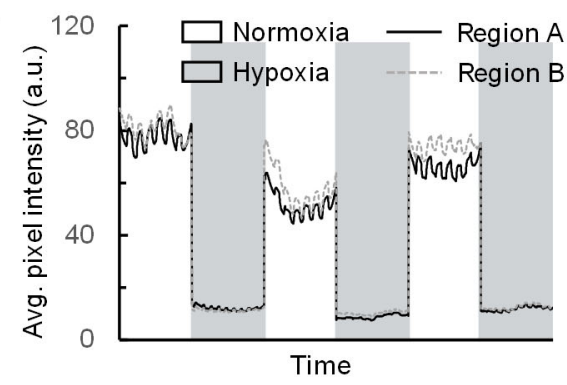

e
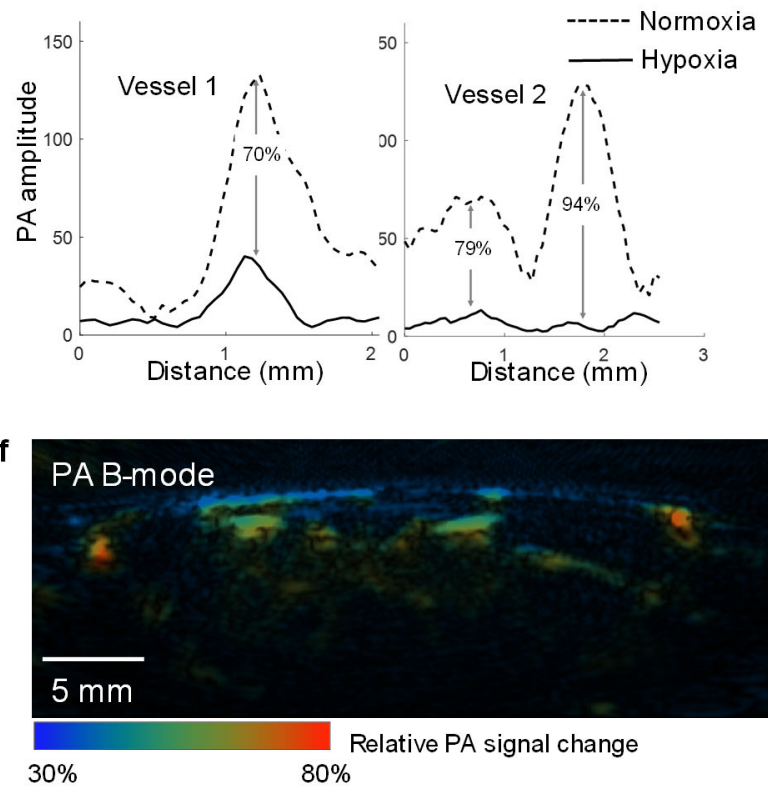

b
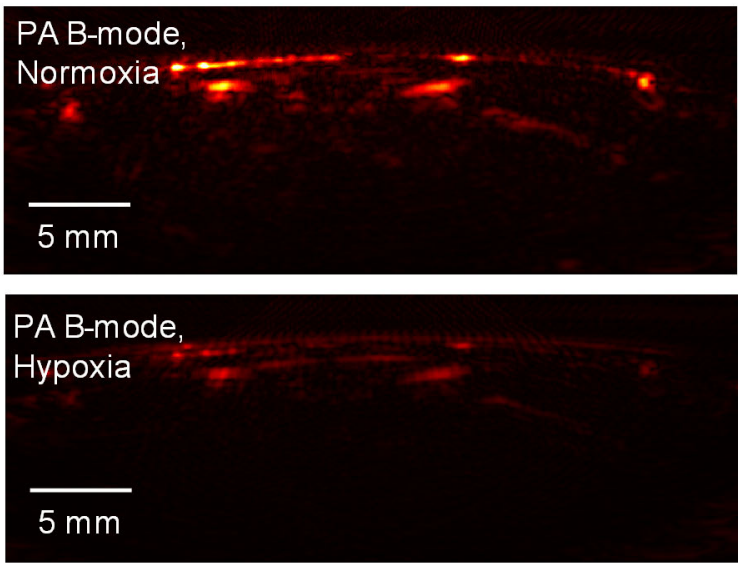

C
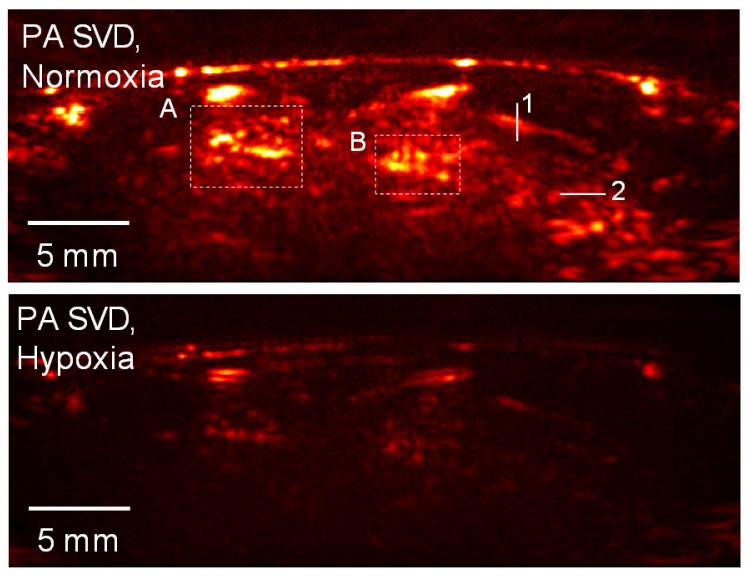

Min $\quad \operatorname{Max}$ PA amplitude (a.u.)

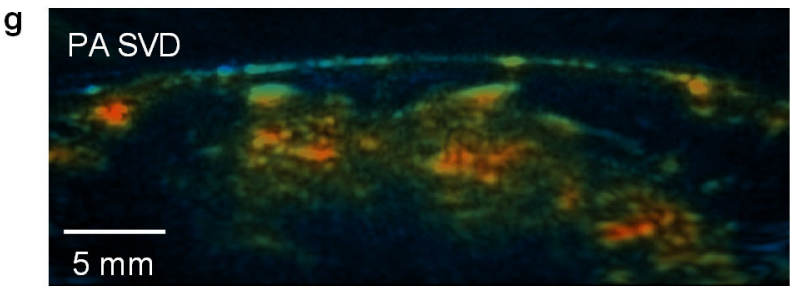

Figure 7: Functional VPS-PAT of rat liver under hypoxia challenge. (a) Normoxia and hypoxia were alternated for 3 cycles, with $21 \%$ and $10 \%$ oxygen level supplied to normoxia and hypoxia respectively. (b) B-mode PA images under normoxia and hypoxia conditions. (c) SVD-filtered PA images under normoxia and hypoxia conditions. (d) Representative regional SVD-filtered signal intensity as a function of time for normoxia and hypoxia sessions. The two regions are identified by the dashed box in (c). (e) Representative vessel signal profiles under normoxia and hypoxia conditions. The two vessels are indicated by the solid 
lines in (c). (f-g) The relative signal changes from normoxia to hypoxia, extracted from the B-mode images (f) and SVD-filtered images (g). 


\section{Supplementary Files}

This is a list of supplementary files associated with this preprint. Click to download.

- Supplementaryvideoandfigurev4.pdf

- supv1annotated.mp4

- supv2annotated.mp4

- supv3annotated.mp4

- supv4annotated.mp4

- supv5annotated.mp4

- supv6annotated.mp4

- supv7annotated.mp4

- supv8annotated.mp4

- supv9annotated.mp4

- supv10.mp4 